\title{
Deep Denoising of Flash and No-Flash Pairs for Photography in Low-Light Environments
}

\author{
Zhihao Xia ${ }^{1}$, Michaël Gharbi ${ }^{2}$, Federico Perazzi $^{3}$, Kalyan Sunkavalli $^{2}$, Ayan Chakrabarti $^{1}$ \\ ${ }^{1}$ Washington University in St. Louis $\quad{ }^{2}$ Adobe Research $\quad{ }^{3}$ Facebook \\ \{zhihao.xia, ayan\}@wustl.edu, \{mgharbi, sunkaval\}@adobe.com, fperazzi@fb.com
}

\begin{abstract}
We introduce a neural network-based method to denoise pairs of images taken in quick succession, with and without a flash, in low-light environments. Our goal is to produce a high-quality rendering of the scene that preserves the color and mood from the ambient illumination of the noisy no-flash image, while recovering surface texture and detail revealed by the flash. Our network outputs a gain map and a field of kernels, the latter obtained by linearly mixing elements of a per-image low-rank kernel basis. We first apply the kernel field to the no-flash image, and then multiply the result with the gain map to create the final output. We show our network effectively learns to produce high-quality images by combining a smoothed out estimate of the scene's ambient appearance from the no-flash image, with high-frequency albedo details extracted from the flash input. Our experiments show significant improvements over alternative captures without a flash, and baseline denoisers that use flash no-flash pairs. In particular, our method produces images that are both noise-free and contain accurate ambient colors without the sharp shadows or strong specular highlights visible in the flash image.
\end{abstract}

\section{Introduction}

Flash photography has long been a convenient way to capture high-quality images in low-light conditions. A flash illuminates the scene with a bright burst of light at the time of exposure, allowing the camera to acquire a photograph with a much higher signal-to-noise ratio than would be possible under the dim ambient lighting alone and without introducing any motion or defocus blur. The flash addresses the problem of limited illumination at its root—by adding light to the scene. However, flash illumination is not without drawbacks. An on-camera flash often creates unappealing flat shading and harsh shadows, resulting in images that fail to capture the true mood and ambience of the scene.

Researchers have considered combining pairs of flash
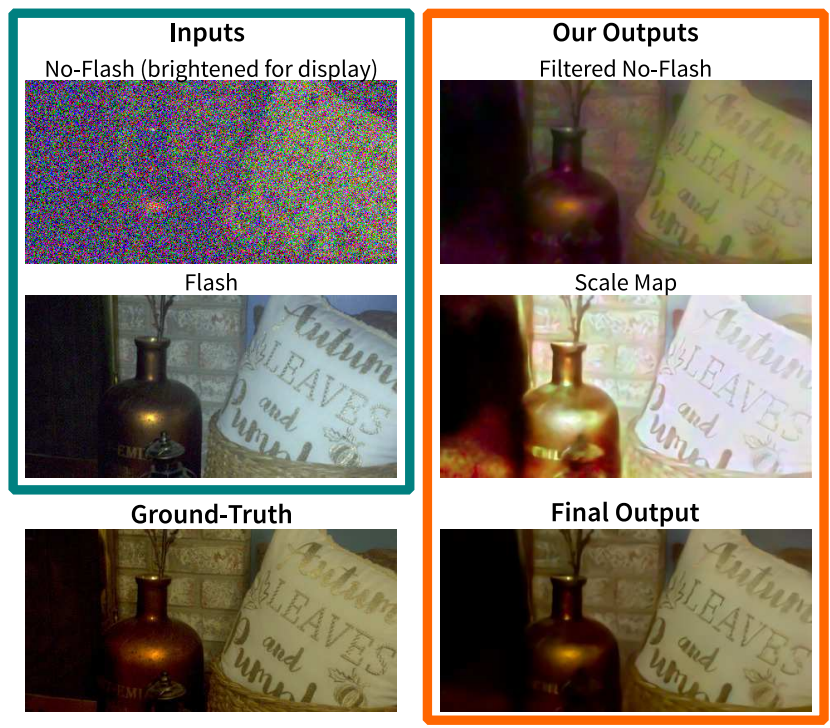

Figure 1: Given a pair of images of low-light scenes captured with and without a flash (left), our method produces a high-quality image of the scene under ambient lighting (right). This output is generated by filtering the no-flash image with a predicted field of kernels - to capture a smoothed stimate of scene appearance under ambient lighting, followed by multiplication with a scale map that introduces high-frequency detail illuminated by the flash.

and no-flash images - captured in quick succession with and without the flash-to create a single enhanced photograph that is both noise-free and accurately represents the scene under ambient lighting. This is achieved by merging information about the ambient scene appearance from the noisy no-flash image, with high-frequency surface image details revealed by the flash $[9,29]$. However, these methods assume moderate levels of noise in the no-flash image, and that the flash and no-flash pair are, or can be, aligned.

In extremely low light, the no-flash image can be very noisy, especially when using mobile phone cameras with small apertures. This precludes the use of traditional 
flash/no-flash methods, since the noise obscures even the low-frequency shading information in the no-flash image and makes automatic alignment of the pair unreliable. In comparison, modern neural network-based denoising methods [5, 34, 40, 41] can produce reasonable estimates from a noisy no-flash image alone-although at high noise-levels, they still struggle to reconstruct high-frequency detail.

In this work, we leverage both the ability of modern neural networks to encode strong natural image priors, and the unique combination of appearance information available in a flash and no-flash image pair. Specifically, we consider the task of producing a high-quality image of the scene under ambient lighting given a flash and no-flash pair as input. We focus on extremely low-light scenes such that the no-flash image shows significant noise, and the appearance of the no-flash image is entirely dominated by the flash illumination. We further assume unknown geometric misalignment between the image pair, due to camera movement typically observed in hand-held burst photography [33].

Under these conditions, we train a deep neural network to take noisy, misaligned no-flash/flash image pairs as input, and output a denoised image of the scene under the scene's ambient illumination. Rather than directly predicting the denoised image, our network outputs a kernel field used to filter the no-flash image, and a scale map that is multiplied with this filtered output to incorporate high-frequency image details from the flash image. To use the regularizing effect of kernels to effectively filter out the high levels of noise in the no-flash input while overcoming its significant memory and computational costs [26], our network combines a recent kernel basis prediction approach [35] with efficient kernel up-sampling. The use of a scale map is inspired by classical flash/no-flash approaches [9, 29] that adopt multiplicative combination based on a view of factorizing images into albedo and shading, where the former is common across the input pair while the latter is not.

We evaluate our approach extensively under different ambient light levels and spatial misalignment, and demonstrate state-of-the-art results for low-light denoising (see example result in Fig. 1). Our method outperforms denoising without a flash-when using a single or burst of two noflash images. This demonstrates that a flash input, despite often representing drastically different shading, is still informative towards ambient appearance. Our method also outperforms other standard denoising approaches trained directly on flash/no-flash pairs, highlighting the importance of the formulation and design of our network architecture.

Code and pre-trained models for our method are available at https://www.cse.wustl.edu/ zhihao.xia/deepfnf/.

\section{Related Work}

Image Denoising. Early works reduced image noise using regularization schemes like sparse-coding [21] and low- rank factorization [13] to model the local statistics of natural images. Other classical approaches have exploited the recurrence of natural image patterns, averaging pixels with similar local neighborhoods [2, 8, 23, 28, 30, 31, 38]. Current state-of the-art denoisers use deep neural networks. Burger et al. [3] were the first to show the ability of even shallow multi-layer perceptrons to to outperform traditional methods such as BM3D [7], and more recent approaches utilizing deeper networks and complex architectures [24, 34, 36, 39, 40, 41] have since led to further improvements in reconstruction accuracy.

Burst Image Denoising. Burst imaging can achieve impressive denoising results, by capturing multiple frames in quick succession. Recent burst denoising algorithms have focused on circumventing the frame misalignment that exists in a real burst. Some methods estimate pixel-wise displacement [14, 15, 16, 19, 25]; others only require coarse global registration, and rely on neural networks to account for the remaining displacement. Amongst the latter group, kernel prediction networks [12, 26, 27] have demonstrated superior ability to recover from misalignment. Our method builds upon Xia et al. [35], which predicts a lowdimensional kernel decomposition, using a linear basis and mixing coefficients, to efficiently realize larger kernels, and to induce regularization leading to improved reconstructions. However, unlike Xia et al. [35] that operate on a burst of images, our approach works on misaligned flash and noflash image pairs, and uses a scale map rather than filtering to extract information from our no-flash input, and an upsampling approach to realize even larger kernels.

Flash Denoising. Flash photography enables the capture of low-noise images in low-light environments using short exposure times and low ISO settings. But, the additional source flash light drastically changes the mood of the scene captured. To remedy this while benefiting from the flash image's higher signal-to-noise ratio, several approaches have used the flash as reference to denoise a noisy ambient (no flash) image. Petschnigg et al. [29] and Eisemann et al. [9] use the flash photo to guide a joint-bilateral filter that denoises the ambient image, transferring high-frequency content from the flash photo. Krishnan and Fergus [20] exploit the correlations between dark flash images and visible light to denoise the ambient image and restore fine details. Yan et al. [37] combine gradients from the flash image with the no-flash image for denoising. These methods all use hand-crafted heuristics to decide which image features to preserve from each of the flash and no-flash inputs.

More recent work [22, 32] have replaced these heuristics with deep neural networks. Li et al. [22] use the (aligned) flash photo as guidance to denoise ambient images. Wang et al. [32] address some of the shortcomings of dark flash photography by adding a stereo RGB image to the capture 


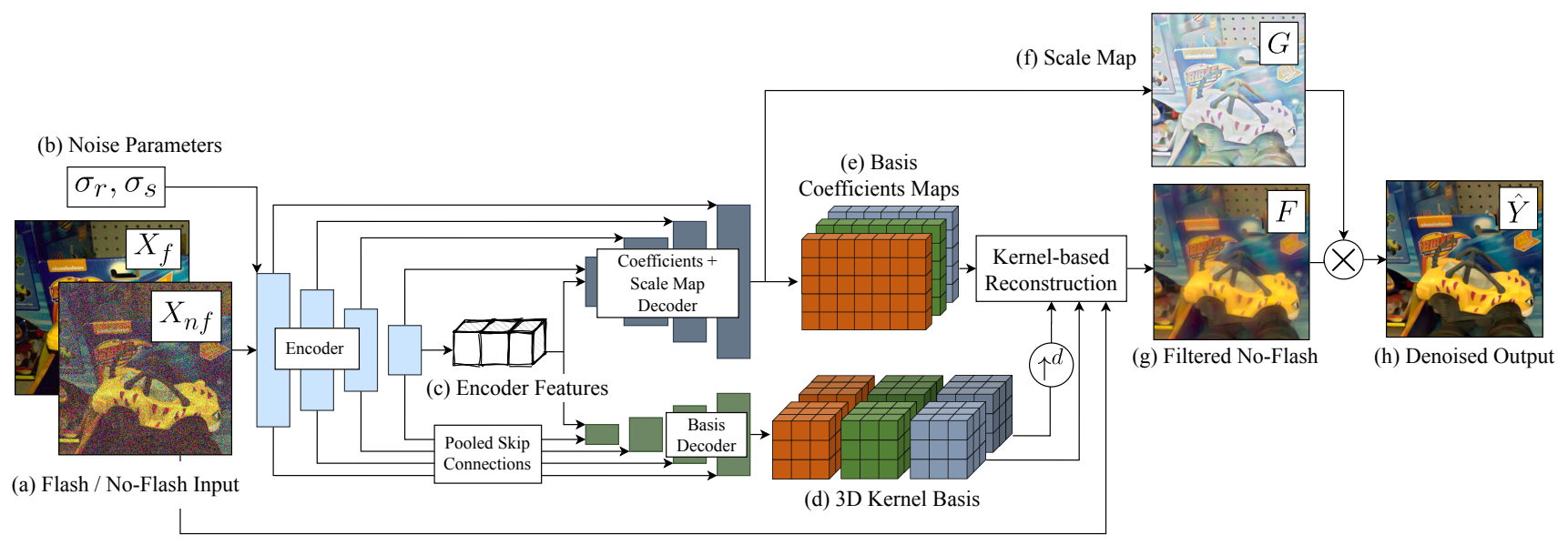

Figure 2: System Overview. The denoising network takes as input a pair of flash, no-flash images (a) together with the noise parameters (b). After encoding, the resulting features (c) are decoded into a multi-scale basis (d), a set of pixel-wise coefficients (e) and a scale map (f). The no-flash image is filtered using the reconstructed kernels (g) and multiplied by the scale map to produce the final denoised output (h).

setup. After being registered and aligned the two images are fused using recent techniques for hyperspectral image restoration and fast image enhancement [6,11]. Unlike these methods, our approach handles motion between the flash/no-flash pairs and large noise levels by using large, learned denoising kernels robust to misalignment.

Beyond denoising, flash photography has also been used for other applications such as deblurring [42], shape estimation [4], and to separate shading from different ambient illuminants present in a scene [17].

\section{Proposed Approach}

Our goal is to estimate a noise-free color image $Y[n] \in$ $\mathbb{R}^{3}$ of the scene under ambient illumination, from a pair of flash and no-flash images $X_{f}[n] \in \mathbb{R}^{3}$ and $X_{n f}[n] \in \mathbb{R}^{3}$, where $n \in \mathbb{Z}^{2}$ denotes the pixel location. Since both images are of the same scene, they represent observations of the same surfaces, with the same material properties, but under different illuminations, and with a potential change in viewpoint due to hand motion between the two shots.

\subsection{Observation Model and Problem Formulation}

In a chosen reference frame, we denote the appearance of the scene under ambient-only illumination as $S_{a}[n] \in \mathbb{R}^{3}$, and under flash-only illumination as $S_{f}[n] \in \mathbb{R}^{3}$. Further, we model the geometric transformations from the reference to the flash and no-flash images as $2 \mathrm{D}$ warps $\mathcal{T}_{f}(n)$ and $\mathcal{T}_{n f}(n)$, respectively. Then, the noise-free versions $\tilde{X}_{n f}[n]$ and $\tilde{X}_{f}[n]$ of our no-flash and flash inputs are given by:

$$
\begin{aligned}
\tilde{X}_{n f}\left[\mathcal{T}_{n f}(n)\right] & =S_{a}[n], \\
\tilde{X}_{f}\left[\mathcal{T}_{f}(n)\right] & =\alpha_{f}\left(S_{f}[n]+S_{a}[n]\right),
\end{aligned}
$$

where $\alpha_{f} \leq 1$ is a scalar that captures the effect of a possibly shorter exposure time for the flash image. Note that since the flash is typically much brighter than the ambient lighting $\left(S_{f}[n] \gg S_{a}[n]\right)$, the contribution of the flash-only appearance is dominant in the flash image $\tilde{X}_{f}[n]$.

As in [27, 35], we assume a heteroscedastic Gaussian noise model [10] to account for both read and shot sensor noise. The observed input flash and no-flash pair relate to their ideal noise-free version (Eq. (1)) as:

$$
\begin{aligned}
X_{f}[n] & \sim \mathcal{N}\left(\tilde{X}_{f}[n], \sigma_{r}^{2}+\sigma_{s}^{2} \tilde{X}_{f}[n]\right), \\
X_{n f}[n] & \sim \mathcal{N}\left(\tilde{X}_{n f}[n], \sigma_{r}^{2}+\sigma_{s}^{2} \tilde{X}_{n f}[n]\right),
\end{aligned}
$$

where $\sigma_{r}^{2}, \sigma_{s}^{2}$ are read and shot noise parameters, which we assume are known. Given $X_{f}[n]$ and $X_{n f}[n]$, and the values of $\sigma_{r}^{2}$ and $\sigma_{s}^{2}$, we seek to estimate $Y[n]:=S_{a}[n]$.

Flash vs. No-flash as reference. Note that in formulation above, we make a distinction between the target output $Y[n]$ and the noise-free no-flash image $\tilde{X}_{n f}[n]$, because they differ by the warp $T_{n f}(n)$. We may wish to use either of the two inputs (flash or no-flash) as the geometric reference. If for instance, the no-flash image is the reference, we assume $T_{n f}(n)=n$ is the identity transformation, and $Y[n]=\tilde{X}_{n f}[n]$. Conversely, if we choose the flash image as reference, $T_{f}(n)=n$ is choosen to be the identity mapping. In Section 4, we analyze the effect of this design choice on the output image quality, finding that in most settings, the choice of the no-flash image as reference yields more accurate reconstructions on average.

\subsection{Enhancement Network}

We use the basis prediction approach of Xia et al. [35], which was designed for burst denoising, as the starting point 
for our model design. Our network differs in two crucial aspects: (a) rather than predicting kernels to filter both the flash and no-flash inputs and summing the result, we filter only the no-flash image and multiply a predicted per-pixel three-channel scale map to form our final output; and (b) we propose an efficient approach to predict larger kernels through upsampling, which is necessary in our setting, because we are filtering a single, highly noisy image. We show an overview of our approach in Figure 2, and include a complete description of our network in the supplement.

\subsubsection{Input data}

Our network takes a twelve channel tensor as input, with six channels containing the observed flash $X_{f}$ and no-flash $X_{n f}$ pair (Equation 2) themselves, and another six encoding the expected per-pixel standard deviation of noise in these inputs, computed using the (known) values of $\sigma_{s}^{2}$ and $\sigma_{r}^{2}$ and the observed noisy intensities as: $\sqrt{\sigma_{r}^{2}+\sigma_{s}^{2} \max \left(0, X^{i}[n]\right)}$, for each channel $i \in\{R, G, B\}$ and $X=X_{f}$ and $X_{n f}$.

\subsubsection{Predicting a global kernel basis}

Like [35], our network features a common encoder whose output is fed to two decoders. The first decoder outputs a global low-rank kernel basis. Unlike [35], we do not constrain our kernels to be positive and unit-normalized. The second decoder outputs per-pixel mixing coefficients to combine the predicted basis elements and form per-pixel kernels. Another departure from [35], the second decode also outputs a 3-channel scale map. We include skipconnections from the encoder to both decoders, using global pooling for connections to the basis decoder as in [35].

\subsubsection{Large kernels by interpolation}

A key innovation in our method over [35] is that our basis encodes larger kernels using a 2-scale representation and an interpolation-based reconstruction scheme. This is crucial in our application where these kernels are used to smooth only one image - the noisy no-flash input-rather than a burst of images as in [35].

Specifically, our basis decoder outputs a set of $J$ basis elements, each consisting of a pair of three-channel kernels $\left\{\left(A_{j}, B_{j}\right)\right\}_{j=1}^{J}$, where each $A_{j}, B_{j} \in \mathbb{R}^{K \times K \times 3}$. We interpret the second kernel $B_{j}$ of each pair as a low-frequency term: a large kernel downsampled by a factor $d$, with an effective $((K-1) * d+1) \times((K-1) * d+1)$ footprint. The $j^{t h}$ element of our basis is then given by $A_{j}+\left(B_{j} \uparrow^{d}\right)$, where $\uparrow^{d}$ denotes bilinear upsampling by a factor $d$. So that $A_{j}$ can add fine high-frequency details to the kernel center. In our experiments, we use a basis with $J=90$ kernels, with a base size $K=15$ and upsampling factor of $d=4$ resulting in an effective kernel size of $57 \times 57$.

\subsubsection{Final reconstruction}

Denoting the per-pixel coefficients from the second decoder as $\left\{c_{j}[n]\right\}_{j=1}^{J}$, we first filter the no-flash input image as:

$$
F[n]=\sum_{j=1}^{J} c_{j}[n]\left(X_{n f} *\left(A_{j}+B_{j} \uparrow^{d}\right)\right)[n] .
$$

where $*$ denotes per-channel convolution between threechannel images and kernels. Note that the filtering with upsampled kernels can be carried out efficiently, by prefiltering the no-flash image and using dilated convolutions:

$$
F[n]=\sum_{j=1}^{J} c_{j}[n]\left(\left(X_{n f} * A_{j}\right)[n]+\left(X_{n f}^{h} *_{d} B_{j}\right)[n]\right),
$$

where $X_{n f}^{h}[n]=\left(X_{n f} * h\right)[n]$ is the result of smoothing the no-flash input with a $(2 d-1) \times(2 d-1)$ tent kernel $h[n]$, and $*_{d}$ represents dilated convolution with a factor of $d$.

Recovering high-frequency detail with a scale map. The result $F[n]$ of this filtering step will typically encode a noise-free (and in the case of the flash as reference, an aligned) estimate of scene appearance under ambient illumination. However, due to the lower signal-to-noise ratio of $X_{n f}$, this filtering step cannot recover the high-frequency details that are illuminated only resolved in the flash image. To recover these, our full-pixel decoder also produces a scale map $G[n] \in \mathbb{R}^{3}$. Our final output $\hat{Y}[n]$ is given by the element-wise product of this scale map and the filtered no-flash image:

$$
\hat{Y}[n]=F[n] \odot G[n] .
$$

This formulation is inspired by classic flash/no-flashing denoising methods [9, 29] that add high-frequency details from the flash image in the log domain, i.e., corresponding to a product in our linear domain. In Section 4, we show this outperforms the alternative of using kernels to jointly denoise the no-flash and flash images.

\subsection{Training details}

While our network accepts raw linear sensor measurements as input and produces an estimate of linear intensities in $Y[n]$, it is trained to maximize image quality in a color and gamma-corrected sRGB space. In particular, we assume that for each training sample $\left(X_{f}^{t}, X_{n f}^{t}, Y^{t}\right)$, we also have a scalar gain $\alpha^{t}$ (representing a desired target brightness level), and a $3 \times 3$ color transform matrix $C^{t}$ based on camera sensor parameters and white-balance settings, 
such that the mapping to sRGB is given by $f_{t}(Y[n])=$ $\gamma\left(\alpha C^{t} \hat{Y}[n]\right)$, where $\gamma(\cdot)$ is a gamma correction curve.

We train our model to minimize the sum of a squared $L_{2}$ pixel loss, and a $L_{1}$ gradient loss between the estimated and ideal rendered images:

$$
\begin{gathered}
L=\frac{1}{T} \sum_{t=1}^{T}\left\|f_{t}\left(\hat{Y}_{t}\right)-f_{t}\left(Y_{t}\right)\right\|^{2}+\eta\left|\partial_{x} *\left(f_{t}\left(\hat{Y}_{t}\right)-f_{t}\left(Y_{t}\right)\right)\right| \\
+\eta\left|\partial_{y} *\left(f_{t}\left(\hat{Y}_{t}\right)-f_{t}\left(Y_{t}\right)\right)\right|,
\end{gathered}
$$

where $\partial_{x}$ and $\partial_{y}$ are horizontal and vertical gradient filters.

We train our model using the Adam optimizer [18], beginning with a learning rate of $10^{-4}$, and going through two learning rate drops every time validation loss saturates, for a total of roughly 1.5 million iterations.

\section{Experiments}

We now describe experiments evaluating our approach, and comparing it to baseline methods for both denoising without a flash input, and to applying existing network architectures to a flash and no-flash pair. We also include ablations describing the effect of our kernel interpolation approach, and of choosing the no-flash vs. flash image as geometric reference.

\subsection{Preliminaries}

Dataset. We use the dataset of Aksoy et al. [1], which contains 16-bit well-exposed ambient-only and flash-only image pairs. These images were crowdsourced from users who were asked to capture images with hand-held mobile phones in real-world settings, and roughly had a 0.5-1 second delay between captures of the pair. We split the dataset as follows: 2519 images for the training set, 128 for validation and 128 for testing, considering $440 \times 440$ crops (random crops for training, and fixed central crops for validation and testing). We simulate a real low-light capture by dimming the linear ambient-only image by dividing with a random factor in $[2,50]$, sampled uniformly in the log domain. This forms our no-flash input. We increase the exposure of the flash-only image by a constant factor 2 , and add it to the no-flash input to obtain our flash input.

Misalignment and simulated noise. The image pairs provided by [1] were automatically aligned by finding correspondences with feature matching. Since this would be unrealistic in low-light images, we undo such alignment by warping the no-flash or flash image (the other is the reference) with a random homography. To obtain the homography parameters, we assume the camera's FOV is 90 degrees to get its intrinsic matrix. We perturb it with a random 3D rotation uniformly sampled in the range $[-0.5,0.5]$ degrees in each axis, followed by random $2 \mathrm{D}$ scaling by a factor uniformly sampled in $[0.98,1.02]$, and a random $2 \mathrm{D}$ translation of $[0,2]$ pixels. The overall average per-pixel displacement between our flash and no-flash inputs ranges up to 20 pixels (Manhattan distance). Note that real-world non-idealities like parallax, occlusions, blur, etc. originally present in the data are preserved by undoing [1]'s alignment.

We use the same noise parameters for the flash and noflash image, i.e., we assume they were captured with the same ISO setting. During training, we randomly sample the noise parameters $\sigma_{r}$ and $\sigma_{s}$ uniformly in the log-domain in the ranges: $\log \left(\sigma_{r}\right) \in[-3,-2]$ and $\log \left(\sigma_{s}\right) \in[-4,-2.6]$.

Losses and metrics. The preprocessing pipeline is executed on the original linear color space of the camera. To compute losses, we set the desired gain $\alpha^{t}$ in Sec. 3.3 to be the inverse of the factor we used to dim the image above, since the original images in [1] were well-lit. The database also includes a color transform matrix for each image which we use as $C^{t}$. We evaluate performance by computing PSNR and SSIM between the rendered versions of our estimate and the ground-truth.

Baselines. We compare to denoising without a flash input: using a single no-flash image denoised by a version of our architecture (without a scale map), and a burst of two (misaligned) no-flash inputs denoised using the stateof-the-art burst denoising method of [35] (which we refer to as BPN). For flash and no-flash image inputs, we compare our method to other standard architectures: a direct prediction network which simply regresses to the denoised output, and burst denoising methods KPN [27] and BPN [35] applied to the flash and no-flash pair. All of these methods were trained on our dataset, and provided information about noise standard deviation in an identical manner to our method. Further details are included in the supplement.

\subsection{Evaluation}

We begin by evaluating our method, choosing the ambient image as geometric reference (i.e., we assume $T_{n f}(n)=$ $n$ ), on our test set of 128 images, and comparing it to the various baselines described above. We fix the noise level to $\log \left(\sigma_{r}\right)=-2.6$ and $\log \left(\sigma_{s}\right)=-3.6$, sample a random homography for each pair to be applied to the flash image (for the no-flash burst, this homography is applied to the second no-flash image), and repeat our evaluation with a discrete set of dimming factors: $[100,50,25,12.5]$. Note that the factor 100 lies outside our training range, and demonstrates the robustness of our method.

Our method consistently outperforms all methods, regardless of the dimming factor, as seen by the quantitative results in Table 1. We also include example reconstructions in Fig. 4, where we see that our method reconstructs fine surface detail with higher fidelity than the other methods.

In Fig. 3, we take a closer look at the effect of misalignment. We take a subset of 64 flash and no-flash pairs 


\begin{tabular}{|c|c|c|c|c|c|c|c|c|}
\hline \multirow{2}{*}{ Method } & \multicolumn{2}{|c|}{ 100x Dimmed } & \multicolumn{2}{|c|}{ 50x Dimmed } & \multicolumn{2}{|c|}{ 25x Dimmed } & \multicolumn{2}{|c|}{ 12.5x Dimmed } \\
\hline & PSNR & SSIM & PSNR & SSIM & PSNR & SSIM & PSNR & SSIM \\
\hline \multicolumn{9}{|l|}{ No-flash input only } \\
\hline Our Architecture & $24.91 \mathrm{~dB}$ & 0.779 & $27.23 \mathrm{~dB}$ & 0.825 & $29.31 \mathrm{~dB}$ & 0.865 & $30.98 \mathrm{~dB}$ & 0.895 \\
\hline \multicolumn{9}{|c|}{ 2-frame burst input (no flash) } \\
\hline BPN [35] & $25.58 \mathrm{~dB}$ & 0.796 & $27.75 \mathrm{~dB}$ & 0.839 & $29.65 \mathrm{~dB}$ & 0.874 & $31.21 \mathrm{~dB}$ & 0.899 \\
\hline \multicolumn{9}{|c|}{ Flash and no-flash input pair } \\
\hline Direct Prediction & $24.80 \mathrm{~dB}$ & 0.773 & $27.06 \mathrm{~dB}$ & 0.818 & $29.12 \mathrm{~dB}$ & 0.857 & $30.84 \mathrm{~dB}$ & 0.888 \\
\hline KPN [27] & $25.87 \mathrm{~dB}$ & 0.815 & $27.94 \mathrm{~dB}$ & 0.852 & $29.69 \mathrm{~dB}$ & 0.880 & $31.21 \mathrm{~dB}$ & 0.901 \\
\hline BPN [35] & $26.11 \mathrm{~dB}$ & 0.815 & $28.04 \mathrm{~dB}$ & 0.850 & $29.75 \mathrm{~dB}$ & 0.880 & $31.21 \mathrm{~dB}$ & 0.901 \\
\hline Ours & $26.75 \mathrm{~dB}$ & 0.829 & $28.56 \mathrm{~dB}$ & 0.860 & $30.14 \mathrm{~dB}$ & 0.884 & $31.52 \mathrm{~dB}$ & 0.903 \\
\hline
\end{tabular}

Table 1: Quantitative results. Thanks to the richer signal provided by the flash input, our method outperforms our single image denoising baseline, and a 2-frame burst denoising baseline. Comparisons to standard burst denoising approaches adapted to use flash-no-flash pairs show that our model architecture with its filtering/scale decomposition and larger kernels outperforms previous work. These results hold over a wide range of ambient light levels, shown here as dimming factors between the low-light no-flash input and a well-lit ground-truth target.

\begin{tabular}{rccc}
\hline Setting & $\begin{array}{c}\text { Flash } \\
\text { Reference }\end{array}$ & $\begin{array}{c}\text { No-Flash } \\
\text { Reference }\end{array}$ & $\begin{array}{c}\text { No-Flash } \\
\text { w/o }\left\{B_{j}\right\}\end{array}$ \\
\hline 100x dimmed & $\mathbf{2 6 . 8 3 ~ d B}$ & $26.75 \mathrm{~dB}$ & $26.45 \mathrm{~dB}$ \\
50x dimmed & $28.39 \mathrm{~dB}$ & $\mathbf{2 8 . 5 6} \mathrm{dB}$ & $28.42 \mathrm{~dB}$ \\
25x dimmed & $29.55 \mathrm{~dB}$ & $\mathbf{3 0 . 1 4} \mathbf{d B}$ & $30.09 \mathrm{~dB}$ \\
12.5x dimmed & $30.45 \mathrm{~dB}$ & $\mathbf{3 1 . 5 2} \mathbf{d B}$ & $31.51 \mathrm{~dB}$ \\
\hline
\end{tabular}

Table 2: Ablation study. We compare the performance of our method to two ablations. One uses the flash image instead of the no-flash image as reference for the geometric transformation. The other uses a kernel basis without interpolation, leading to an effective kernel size of only $15 \times 15$.

from our test set (all dimmed with a factor of 50), and evaluate each set with different homographies that cause different average pixel displacements. We plot the PSNR of reconstruction by various methods for different degrees of displacement (for the single no-flash input baseline, these numbers are the same for all displacements). As expected, the accuracy of all methods decreases with greater misalignment. Nevertheless, we find that our method consistently outperforms all baselines, including the single no-flash input even with misalignment greater than 10 pixels.

Additional results, including results on different noise levels and combining flash denoising with burst denoising, can be found in the supplemental material.

\subsection{Ablation}

Section 3.1 considered two options for the alignment reference: with the output geometrically aligned with the flash input, or the no-flash image. In the previous section, we re-

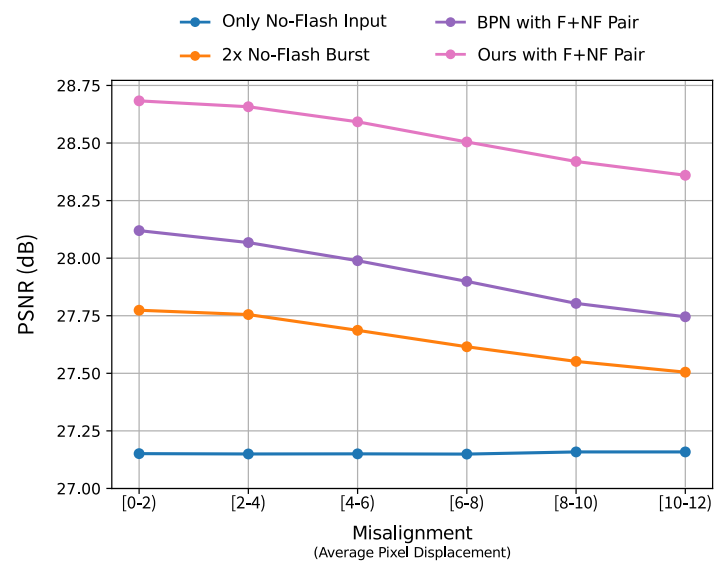

Figure 3: Performance vs. misalignment. We show the performance profile of our method and select baselines as a function of average displacement between the two frames. Our model consistently delivers superior performance and is robust to large misalignment between its inputs.

ported results with the no-flash input as the reference (for our method, as well as the other methods evaluated on the flash and no-flash pair). This was based on an evaluation of both alternatives, which we report in Table. 2 .

We found that except for the lowest light level, the using the no-flash image as reference yields results that are quantitatively better (this is also true for the other baselines). However, looking at the actual reconstructions in Fig. 5, we find both images to be of similar visual quality-with the lower quantitative performance of the flash reference being largely due to slight, and largely imperceptible, alignment errors in low-frequency shading. However, as shown in ex- 


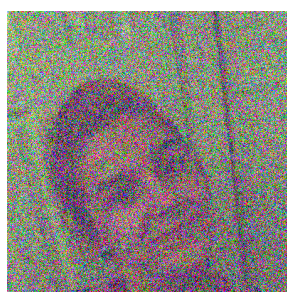

No-Flash Input

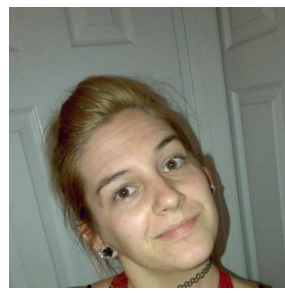

Flash Input

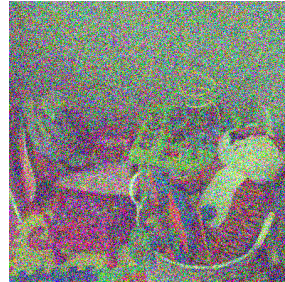

No-Flash Input

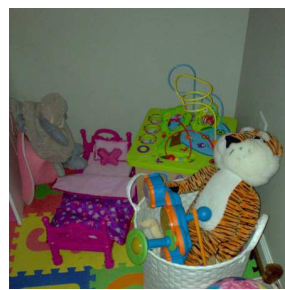

Flash Input

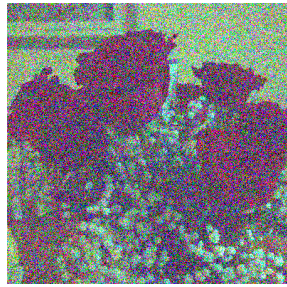

No-Flash Input

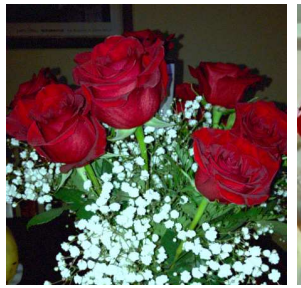

Flash Input

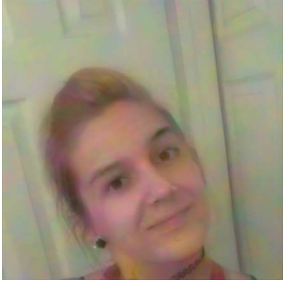

Our Filtered No-Flash

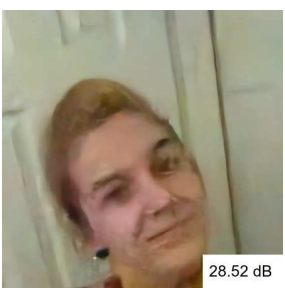

Only No-Flash Input

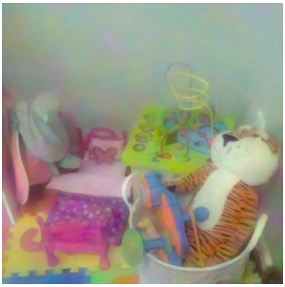

Our Filtered No-Flash

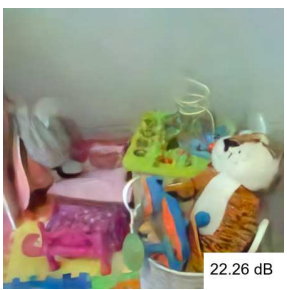

Only No-Flash Input

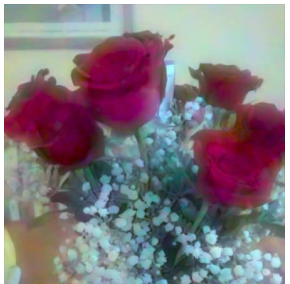

Our Filtered No-Flash

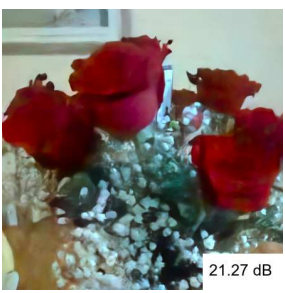

Only No-Flash Input

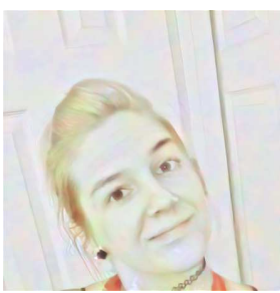

Our Scale Map

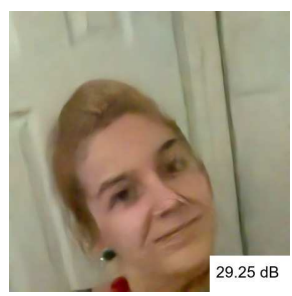

2x No-Flash Burst

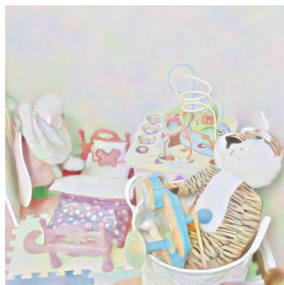

Our Scale Map

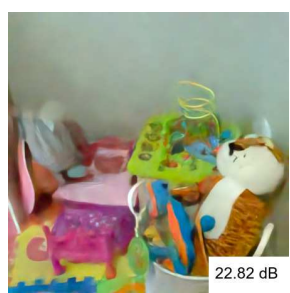

2x No-Flash Burst

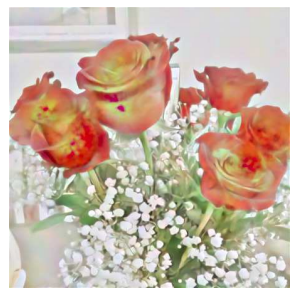

Our Scale Map

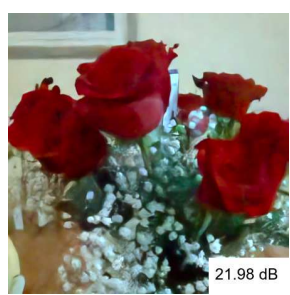

2x No-Flash Burst

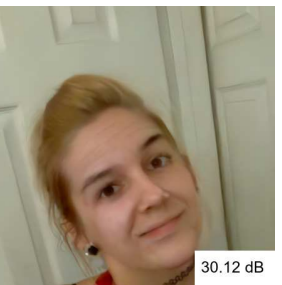

Our Final Output

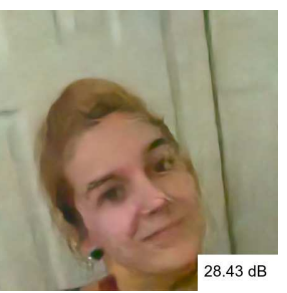

Direct $(F+N F)$

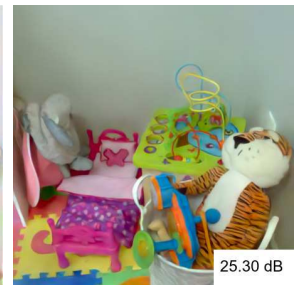

Our Final Output

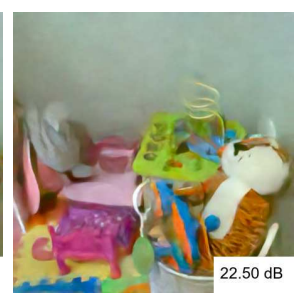

Direct $(\mathrm{F}+\mathrm{NF})$

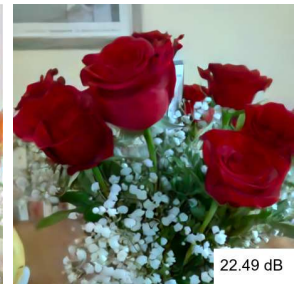

Our Final Output

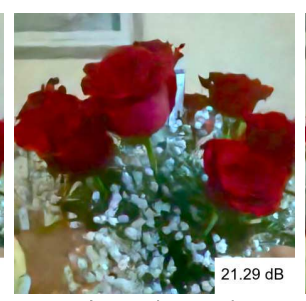

Direct $(\mathrm{F}+\mathrm{NF})$

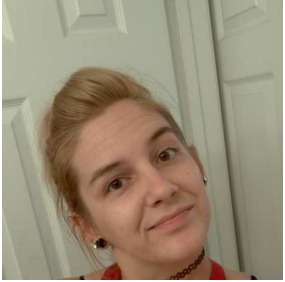

Ground-Truth

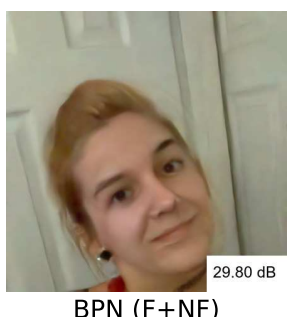

$\mathrm{BPN}(\mathrm{F}+\mathrm{NF})$

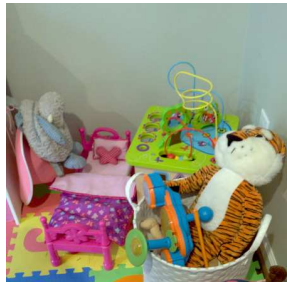

Ground-Truth

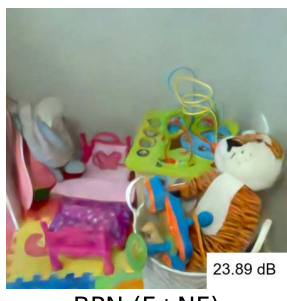

BPN $(F+N F)$

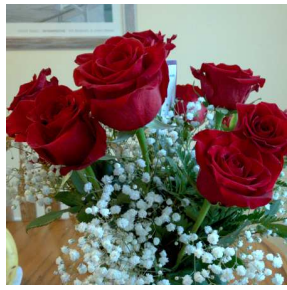

Ground-Truth

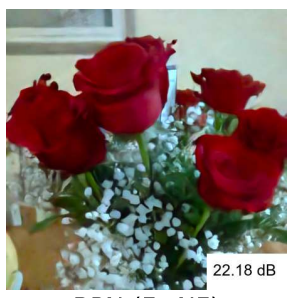

$\mathrm{BPN}(\mathrm{F}+\mathrm{NF})$

Figure 4: Qualitative comparison. Our method uses flash/no-flash image pairs to denoise low-light images. It produces cleaner outputs than baseline flash/no-flash denoisers (Direct $(F+N F), B P N(F+N F)$ ), as well as single-image (Only No-Flash Input $)$ and burst denoisers $(2 \times$ No-Flash Burst $)$. We also visualize our intermediate filtered no-flash image and scale map. 


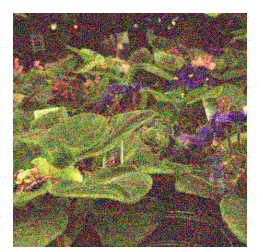

No-Flash Input
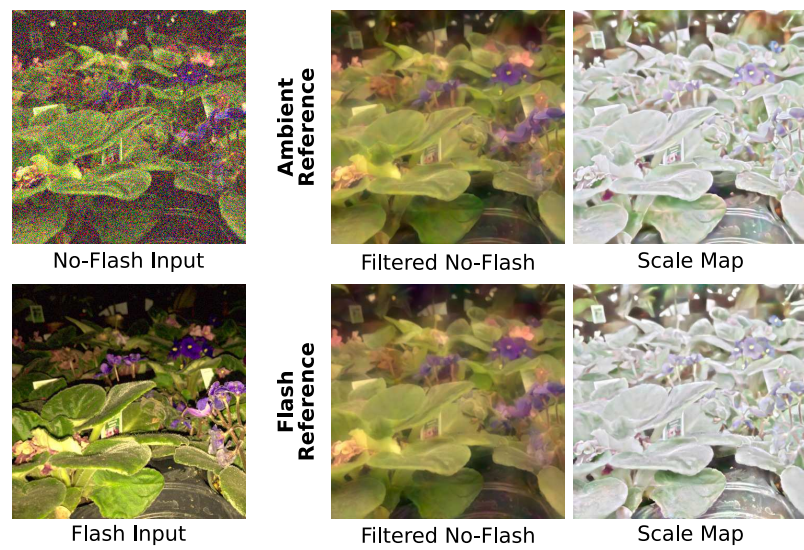

Scale Map
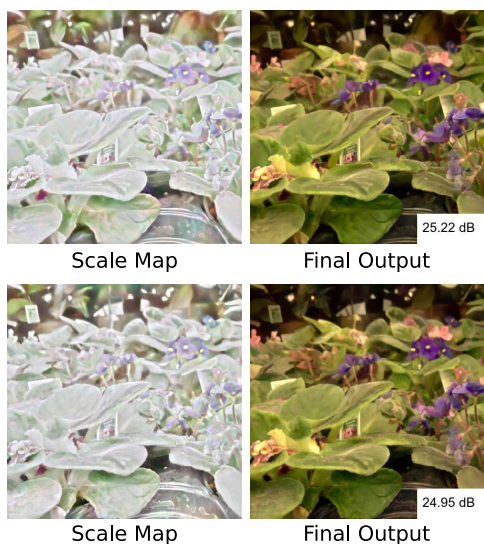

Final Output
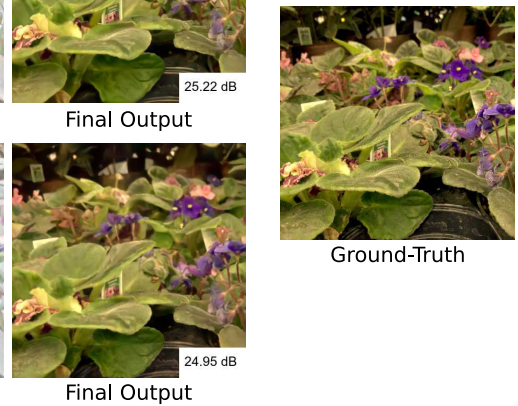

Figure 5: Flash vs. no-flash as reference frame. We use the ambient-only image as the reference frame for our reconstruction (top), i.e. the ground truth is aligned to the no-flash image. We found this choice leads to a lower error on average, compared to the alternative, using the flash as reference (bottom).

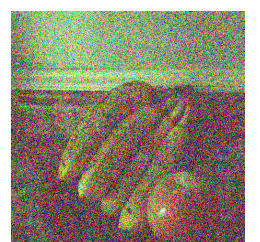

No-Flash Input

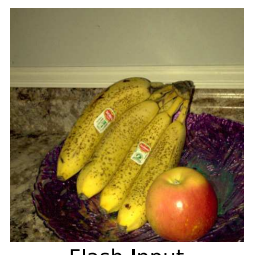

Flash Input

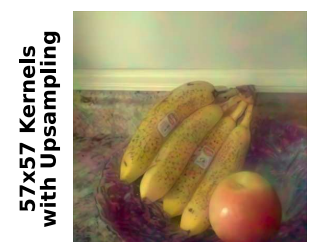

Filtered No-Flash

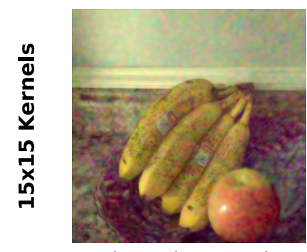

Filtered No-Flash

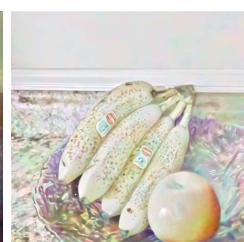

Scale Map

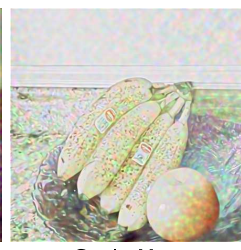

Scale Map

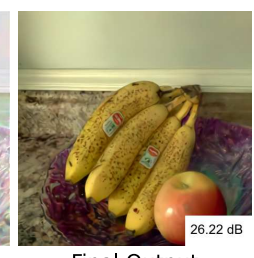

Final Output

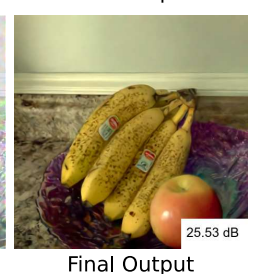

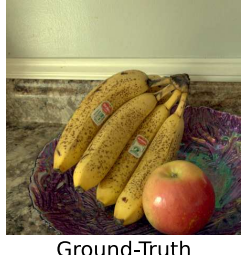

Ground-Truth

Figure 6: Benefit of large kernels. By using a 2-scale kernel decomposition, where the low-pass component is bilinearly upsampled, our model (top) can better denoise the ambient-only image. This leads to reduced residual chroma noise, which makes the scale map more effective at recovering fine details. Without it (bottom), the kernels are too small to effectively denoise the ambient image, so the scale map needs to compensate for the residual mid-frequency noise.

panded analysis in the supplement, using the flash image as reference sometimes yields visually sharper results

Table 2 also evaluates the benefit of using larger filters though our interpolation-based approach. We find that by allowing filters with a larger footprint $(57 \times 57)$, our twoscale kernel basis improves denoising quality, especially at low light levels. As show in Figure 6, large kernels yield a smoother filtering of the noisy no-flash image, so that the flash-driven scale map does not need to overcompensate for residual mid-frequency color noise, leading to better reconstructions in the final output.

\section{Conclusion}

This paper introduced a method to effectively leverage the unique mix of visual information available in a flash and no-flash image pair, and produce high-quality images in low-light environments. Our method preserves the warmth and colors of the ambient lighting while bringing out fine details thanks to the flash image. Drawing on traditional flash/no-flash techniques, our network architecture assembles its output from a filtered ambient-only image, and a scale map that encoded high-frequency details from the flash. Although it was not trained with any intermediate supervision, we found our network automatically learns to carry out both the necessary geometric alignment between the frames, and the photometric transfer needed to produce state-of-the-art reconstructions. Still, there remain situations where flash photography may be too obtrusive. Exploring how our model would fare with dark flash imaging $[20,32]$ is an interesting avenue for future research.

Acknowledgments. ZX and AC acknowledge support from NSF award IIS-1820693, and a gift from Adobe Research. 


\section{References}

[1] Yagiz Aksoy, Changil Kim, Petr Kellnhofer, Sylvain Paris, Mohamed A. Elgharib, Marc Pollefeys, and Wojciech Matusik. A dataset of flash and ambient illumination pairs from the crowd. In Proc. ECCV, 2018. 5

[2] Antoni Buades, Bartomeu Coll, and Jean-Michel Morel. A non-local algorithm for image denoising. In Proc. CVPR, 2005. 2

[3] Harold C Burger, Christian J Schuler, and Stefan Harmeling. Image denoising: Can plain neural networks compete with bm3d? In Proc. CVPR, 2012. 2

[4] Xu Cao, Michael Waechter, Boxin Shi, Ye Gao, Bo Zheng, and Yasuyuki Matsushita. Stereoscopic flash and no-flash photography for shape and albedo recovery. In Proc. CVPR, 2020. 3

[5] Chen Chen, Qifeng Chen, Jia Xu, and Vladlen Koltun. Learning to see in the dark. In Proc. CVPR, 2018. 2

[6] Qifeng Chen, Jia Xu, and Vladlen Koltun. Fast image processing with fully-convolutional networks. In Proc. ICCV, 2017. 3

[7] Kostadin Dabov, Alessandro Foi, Vladimir Katkovnik, and Karen Egiazarian. Color image denoising via sparse 3d collaborative filtering with grouping constraint in luminancechrominance space. In Proc. ICIP, 2007. 2

[8] David L. Donoho. De-noising by soft-thresholding. IEEE Transactions on Information Theory, 41(3):613-627, 1995. 2

[9] Elmar Eisemann and Frédo Durand. Flash photography enhancement via intrinsic relighting. ACM Transactions on Graphics (TOG), 23(3):673-678, 2004. 1, 2, 4

[10] Alessandro Foi, Mejdi Trimeche, Vladimir Katkovnik, and Karen Egiazarian. Practical poissonian-gaussian noise modeling and fitting for single-image raw-data. IEEE Transactions on Image Processing, 17(10):1737-1754, 2008. 3

[11] Michaël Gharbi, Jiawen Chen, Jonathan T. Barron, Samuel W. Hasinoff, and Frédo Durand. Deep bilateral learning for real-time image enhancement. ACM Transactions on Graphics (TOG), 36(4):118:1-118:12, 2017. 3

[12] Clément Godard, Kevin Matzen, and Matt Uyttendaele. Deep burst denoising. In Proc. ECCV, 2018. 2

[13] Qiang Guo, Caiming Zhang, Yunfeng Zhang, and Hui Liu. An efficient svd-based method for image denoising. IEEE Transactions on Circuits and Systems for Video Technology, 26(5):868-880, 2016. 2

[14] Samuel W Hasinoff, Dillon Sharlet, Ryan Geiss, Andrew Adams, Jonathan T Barron, Florian Kainz, Jiawen Chen, and Marc Levoy. Burst photography for high dynamic range and low-light imaging on mobile cameras. ACM Transactions on Graphics (TOG), 35(6):192, 2016. 2

[15] Felix Heide, Steven Diamond, Matthias Nießner, Jonathan Ragan-Kelley, Wolfgang Heidrich, and Gordon Wetzstein. Proximal: Efficient image optimization using proximal algorithms. ACM Transactions on Graphics (TOG), 35(4):84, 2016. 2

[16] Felix Heide, Markus Steinberger, Yun-Ta Tsai, Mushfiqur Rouf, Dawid Pająk, Dikpal Reddy, Orazio Gallo, Jing Liu, Wolfgang Heidrich, Karen Egiazarian, et al. Flexisp: A flexible camera image processing framework. ACM Transactions on Graphics (TOG), 33(6):231, 2014. 2
[17] Zhuo Hui, Kalyan Sunkavalli, Sunil Hadap, and Aswin C. Sankaranarayanan. Illuminant spectra-based source separation using flash photography. In Proc. CVPR, 2018. 3

[18] Diederik P Kingma and Jimmy Ba. Adam: A method for stochastic optimization. arXiv preprint arXiv:1412.6980, 2014. 5

[19] Filippos Kokkinos and Stamatis Lefkimmiatis. Iterative residual cnns for burst photography applications. In Proc. CVPR, 2019. 2

[20] Dilip Krishnan and Rob Fergus. Dark flash photography. ACM Transactions on Graphics (TOG), 28(3):96, 2009. 2, 8

[21] Huibin Li and Feng Liu. Image denoising via sparse and redundant representations over learned dictionaries in wavelet domain. In Proc. International Conference on Image and Graphics (ICIG), 2009. 2

[22] Yijun Li, Jia-Bin Huang, Narendra Ahuja, and Ming-Hsuan Yang. Deep joint image filtering. In Proc. ECCV, 2016. 2

[23] Michael Lindenbaum, M. Fischer, and Alfred M. Bruckstein. On gabor's contribution to image enhancement. Pattern Recognit., 27(1):1-8, 1994. 2

[24] Ding Liu, Bihan Wen, Yuchen Fan, Chen Change Loy, and Thomas S Huang. Non-local recurrent network for image restoration. In Advances in Neural Information Processing Systems, pages 1680-1689, 2018. 2

[25] Ziwei Liu, Lu Yuan, Xiaoou Tang, Matt Uyttendaele, and Jian Sun. Fast burst images denoising. ACM Transactions on Graphics (TOG), 33(6):232, 2014. 2

[26] Talmaj Marinč, Vignesh Srinivasan, Serhan Gül, Cornelius Hellge, and Wojciech Samek. Multi-kernel prediction networks for denoising of burst images. In Proc. ICIP, 2019. 2

[27] Ben Mildenhall, Jonathan T Barron, Jiawen Chen, Dillon Sharlet, Ren Ng, and Robert Carroll. Burst denoising with kernel prediction networks. In Proc. CVPR, 2018. 2, 3, 5, 6, ii, vi

[28] Pietro Perona and Jitendra Malik. Scale-space and edge detection using anisotropic diffusion. IEEE Transactions on pattern analysis and machine intelligence, 12(7):629-639, 1990. 2

[29] Georg Petschnigg, Richard Szeliski, Maneesh Agrawala, Michael Cohen, Hugues Hoppe, and Kentaro Toyama. Digital photography with flash and no-flash image pairs. ACM Transactions on Graphics (TOG), 23(3):664-672, Aug. 2004. 1, 2, 4

[30] Leonid I Rudin, Stanley Osher, and Emad Fatemi. Nonlinear total variation based noise removal algorithms. Physica D: nonlinear phenomena, 60(1-4):259-268, 1992. 2

[31] Carlo Tomasi and Roberto Manduchi. Bilateral filtering for gray and color images. In Proc. ICCV, 1998. 2

[32] Jian Wang, Tianfan Xue, Jonathan T. Barron, and Jiawen Chen. Stereoscopic dark flash for low-light photography. In Proc. ICCP, 2019. 2, 8

[33] Bartlomiej Wronski, Ignacio Garcia-Dorado, Manfred Ernst, Damien Kelly, Michael Krainin, Chia-Kai Liang, Marc Levoy, and Peyman Milanfar. Handheld multi-frame superresolution. ACM Transactions on Graphics (TOG), 38(4):118, 2019. 2

[34] Zhihao Xia and Ayan Chakrabarti. Identifying recurring patterns with deep neural networks for natural image denoising. 
In Proc. WACV, 2020. 2

[35] Zhihao Xia, Federico Perazzi, Michaël Gharbi, Kalyan Sunkavalli, and Ayan Chakrabarti. Basis prediction networks for effective burst denoising with large kernels. In Proc. CVPR, 2020. 2, 3, 4, 5, 6, i, ii, vi

[36] Junyuan Xie, Linli Xu, and Enhong Chen. Image denoising and inpainting with deep neural networks. In Advances in Neural Information Processing Systems, pages 341-349, 2012. 2

[37] Qiong Yan, Xiaoyong Shen, Li Xu, Shaojie Zhuo, Xiaopeng Zhang, Liang Shen, and Jiaya Jia. Cross-field joint image restoration via scale map. In Proc. ICCV, 2013. 2

[38] Leonid P Yaroslavsky. Digital picture processing: an introduction. Applied Optics, 25(18):3127, 1986. 2
[39] Kai Zhang, Wangmeng Zuo, Yunjin Chen, Deyu Meng, and Lei Zhang. Beyond a gaussian denoiser: Residual learning of deep cnn for image denoising. IEEE Transactions on Image Processing, 26(7):3142-3155, 2017. 2

[40] Kai Zhang, Wangmeng Zuo, Shuhang Gu, and Lei Zhang. Learning deep cnn denoiser prior for image restoration. In Proc. CVPR, 2017. 2

[41] Yulun Zhang, Kunpeng Li, Kai Li, Bineng Zhong, and Yun $\mathrm{Fu}$. Residual non-local attention networks for image restoration. In Proc. ICLR, 2018. 2

[42] Shaojie Zhuo, Dong Guo, and Terence Sim. Robust flash deblurring. In Proc. CVPR, 2010. 3 


\section{Supplementary Material}

\section{A. Architecture Details}

We describe our network architecture in detail in Table 3. Our architecture follows the encoder with dual decoder architecture of [35], but changes the output of the global decoder to output a basis with the two sets of kernels $\left\{\left(A_{j}, B_{j}\right)\right\}$, each a $K \times K \times 3$ kernel, and the per-pixel decoder outputs a scale map in addition to the kernel coefficients.

\begin{tabular}{|c|c|c|c|}
\hline Name & Input & Layer & Output Size \\
\hline Input & - & - & $\mathrm{H} \times \mathrm{W} \times 12$ \\
\hline \multicolumn{4}{|l|}{ Encoder } \\
\hline Enc-0 & Input & $3 \times 3$ Conv & $\mathrm{H} \times \mathrm{W} \times 64$ \\
\hline Enc-1-A & Enc-0 & $3 \times 3$ Conv & $\mathrm{H} \times \mathrm{W} \times 64$ \\
\hline Enc-1-B & Enc-1-A & $3 \times 3$ Conv & $\mathrm{H} \times \mathrm{W} \times 64$ \\
\hline Enc-1-C & Enc-1-B & 2x2 Stride 2 Max Pool & $H / 2 \times W / 2 \times 64$ \\
\hline Enc-2-A & Enc-1-C & $3 \times 3$ Conv & $\mathrm{H} / 2 \times \mathrm{W} / 2 \times 128$ \\
\hline Enc-2-B & Enc-2-A & $3 \times 3$ Conv & $\mathrm{H} / 2 \times \mathrm{W} / 2 \times 128$ \\
\hline Enc-2-C & Enc-2-B & 2x2 Stride 2 Max Pool & $\mathrm{H} / 4 \times \mathrm{W} / 4 \times 128$ \\
\hline Enc-3-A & Enc-2-C & $3 \times 3$ Conv & $\mathrm{H} / 4 \times \mathrm{W} / 4 \times 256$ \\
\hline Enc-3-B & Enc-3-A & $3 \times 3$ Conv & $\mathrm{H} / 4 \times \mathrm{W} / 4 \times 256$ \\
\hline Enc-3-C & Enc-3-B & $2 \times 2$ Stride 2 Max Pool & $\mathrm{H} / 8 \times \mathrm{W} / 8 \times 256$ \\
\hline Enc-4-A & Enc-3-C & $3 \times 3$ Conv & $\mathrm{H} / 8 \times \mathrm{W} / 8 \times 512$ \\
\hline Enc-4-B & Enc-4-A & $3 \times 3$ Conv & $\mathrm{H} / 8 \times \mathrm{W} / 8 \times 512$ \\
\hline Enc-4-C & Enc-4-B & $2 \times 2$ Stride 2 Max Pool & $\mathrm{H} / 16$ x W/16 x 512 \\
\hline Enc-5-A & Enc-4-C & $3 \times 3$ Conv & $\mathrm{H} / 16 \times \mathrm{W} / 16 \times 1024$ \\
\hline Enc-5-B & Enc-5-A & $3 \times 3$ Conv & $\mathrm{H} / 16 \times \mathrm{W} / 16 \times 1024$ \\
\hline Enc-5-C & Enc-5-B & $2 \times 2$ Stride 2 Max Pool & $\mathrm{H} / 32 \times \mathrm{W} / 32 \times 1024$ \\
\hline Enc-F & Enc-5-C & $3 \times 3$ Conv & $\mathrm{H} / 32 \times \mathrm{W} / 32 \times 1024$ \\
\hline Enc-Out & Enc-F & $3 \times 3$ Conv & $\mathrm{H} / 32 \times \mathrm{W} / 32 \times 1024$ \\
\hline \multicolumn{4}{|c|}{ Global Decoder } \\
\hline GDec-5-A & Bilinear-Up(GP(Enc-Out)) & $3 \times 3$ Conv & $2 \times 2 \times 512$ \\
\hline GDec-5-B & GDec-5-A, GP-R[2x2](Enc-5-B) & $3 \times 3$ Conv & $2 \times 2 \times 512$ \\
\hline GDec-5-C & GDec-5-B & $3 \times 3$ Conv & $2 \times 2 \times 512$ \\
\hline GDec-4-A & Bilinear-Up(GDec-5-C) & $3 \times 3$ Conv & $4 \times 4 \times 256$ \\
\hline GDec-4-B & GDec-4-A, GP-R[4x4](Enc-4-B) & $3 \times 3$ Conv & $4 \times 4 \times 256$ \\
\hline GDec-4-C & GDec-4-B & $3 \times 3$ Conv & $4 \times 4 \times 256$ \\
\hline GDec-3-A & Bilinear-Up(GDec-4C) & $3 \times 3$ Conv & $8 \times 8 \times 256$ \\
\hline GDec-3-B & GDec-3-A, GP-R[8,8](Enc-3-B) & $3 \times 3$ Conv & $8 \times 8 \times 256$ \\
\hline GDec-3-C & GDec-3-B & $3 \times 3$ Conv & $8 \times 8 \times 256$ \\
\hline GDec-2-A & Bilinear-Up(GDec-3-C) & $3 \times 3$ Conv & $16 \times 16 \times 128$ \\
\hline GDec-2-B & GDec-2-A, GP-R[16,16](Enc-2-B) & $3 \times 3$ Conv & $16 \times 16 \times 128$ \\
\hline GDec-2-C & GDec-2-B & $3 \times 3$ Conv & $16 \times 16 \times 128$ \\
\hline GDec-F-A & GDec-2-C & $2 \times 2$ Conv (Valid) & $15 \times 15 \times 128$ \\
\hline GDec-F-B & GDec-F-A & $3 \times 3$ Conv & $15 \times 15 \times 128$ \\
\hline Output: Basis & GDec-F-B & $3 \times 3$ Conv & $15 \times 15 \times(3 * 2 * \mathrm{~J})$ \\
\hline
\end{tabular}

Table 3: Our network architecture. Bi-linear upsampling refers to upsampling the feature map by a factor of 2. GP refers to global average pooling, and GP-R[H', W'] to global average pooling followed by replicating spatially to size H' $\mathrm{x}$ W'. Multiple inputs are concatenated along the channel dimension before being passed to the convolution layer. All convolution layers use same padding unless otherwise specified. 


\begin{tabular}{|c|c|c|c|}
\hline Name & Input & Layer & Output Size \\
\hline \multicolumn{4}{|l|}{ Per-pixel Decoder } \\
\hline PDec-5-A & Bilinear-Up(Enc-Out) & $3 \times 3$ Conv & $\mathrm{H} / 16 \times \mathrm{W} / 16 \times 512$ \\
\hline PDec-5-B & PDec-5-A, Enc-5-B & $3 \times 3$ Conv & $\mathrm{H} / 16 \times \mathrm{W} / 16 \times 512$ \\
\hline PDec-5-C & PDec-5-B & $3 \times 3$ Conv & $\mathrm{H} / 16 \times \mathrm{W} / 16 \times 512$ \\
\hline PDec-4-A & Bilinear-Up(PDec-5-C) & $3 \times 3$ Conv & $\mathrm{H} / 8 \times \mathrm{W} / 8 \times 256$ \\
\hline PDec-4-B & PDec-4-A, Enc-4-B & $3 \times 3$ Conv & $\mathrm{H} / 8 \times \mathrm{W} / 8 \times 256$ \\
\hline PDec-4-C & PDec-4-B & $3 \times 3$ Conv & $\mathrm{H} / 8 \times \mathrm{W} / 8 \times 256$ \\
\hline PDec-3-A & Bilinear-Up(PDec-4-C) & $3 \times 3$ Conv & $\mathrm{H} / 4 \times \mathrm{W} / 4 \times 128$ \\
\hline PDec-3-B & PDec-3-A, Enc-3-B & $3 \times 3$ Conv & $\mathrm{H} / 4 \times \mathrm{W} / 4 \times 128$ \\
\hline PDec-3-C & PDec-3-B & $3 \times 3$ Conv & $\mathrm{H} / 4 \times \mathrm{W} / 4 \times 128$ \\
\hline PDec-2-A & Bilinear-Up(PDec-3-C) & $3 \times 3$ Conv & $\mathrm{H} / 2 \times \mathrm{W} / 2 \times 64$ \\
\hline PDec-2-B & PDec-2-A, Enc-2-B & $3 \times 3$ Conv & $\mathrm{H} / 2 \times \mathrm{W} / 2 \times 64$ \\
\hline PDec-2-C & PDec-2-B & $3 \times 3$ Conv & $\mathrm{H} / 2 \times \mathrm{W} / 2 \times 64$ \\
\hline PDec-1-A & Bilinear-Up(PDec-2-C) & $3 \times 3$ Conv & H x W x 64 \\
\hline PDec-1-B & PDec-1-A, Enc-1-B & $3 \times 3$ Conv & $\mathrm{H} \times \mathrm{W} \times 64$ \\
\hline PDec-1-C & PDec-1-B & $3 \times 3$ Conv & $\mathrm{H} \times \mathrm{W} \times 64$ \\
\hline PDec-F-0 & PDec-1-C & $3 \times 3$ Conv & $\mathrm{H} \times \mathrm{W} \times 64$ \\
\hline PDec-F-1 & PDec-F-0 & $3 \times 3$ Conv & $\mathrm{H} \times \mathrm{W} \times 64$ \\
\hline Output: Coeffs + Scale map & PDec-F-1 & $3 \times 3$ Conv & $\mathrm{H} \times \mathrm{W} \times(\mathrm{J}+3)$ \\
\hline
\end{tabular}

Table 3: (continued) Our network architecture.

The output of the global decoder gives us $J=90$ pairs of kernels $\left\{\left(A_{j}, B_{j}\right)\right\}$, and that of the per-pixel decoder both the coefficients $C[n] \in \mathbb{R}^{J}$ and a scale map $G[n] \in \mathbb{R}^{3}$. As noted in the paper, we set $J=90$ in our experiments.

Baselines. Our baselines use similar architectures to our main method to enable a fair comparison. For the no-flash single image input, we use the above architecture to take only the no-flash image as input (6 channels: 3 for the image itself and 3 for noise deviation maps), and do not output a scale map (i.e., our per-pixel decoder only outputs the $J$ channel coefficient map). For the BPN [35] entries in our table for the 2x burst of no-flash images as well as for flash and no-flash denoising, we use the original architecture from their paper. Specifically, our per-pixel decoder again does not output a scale map, and the two sets of kernels are used to filter the two input images which are then added-unlike our approach which combines the two kernels to create a larger upsampled kernel that is only applied to the ambient, followed by multiplication with the scale map. For the direct prediction and KPN [27], we use this architecture without the global decoder. For direct prediction, the per-pixel decoder just outputs a 3-channel map that is treated as a residual and added to the noisy no-flash input to yield the final denoised output. For KPN, the per-pixel decoder outputs a 150-channel output: these are interpreted as two $5 \times 5$ kernels per color channel, to be applied to the flash and no-flash pairs.

\section{B. Additional Results}

Qualitative Results. We show comparison results on more images in Figure 7.

No-Flash vs. Flash as reference. As noted in the paper, using the no-flash image as the geometric reference leads to better performance at all but the darkest light level. This is true not just for our approach, but also the other baselines we considered for denoising with flash and no-flash image pairs as input. We report the performance of these methods when using flash as reference in Table 4, and find that like in the case of no-flash reference, our approach yields superior reconstructions. Although slightly worse on average, we find that using flash as reference can sometimes lead to superior reconstruction of high-frequency details for some images compared to the no-flash reference, and even when the results are quantitatively worse, this is because of misalignment of low-frequency shading that is often not perceptible. We illustrate this with qualitative comparisons in Fig. 8.

Other Noise Levels. In addition to the original values of read and shot noise variances used in the main results table, Table 5 reports the performance of our method, and other baselines for denoising flash and no-flash pairs, for three additional sets of noise parameters at one of the light levels. 


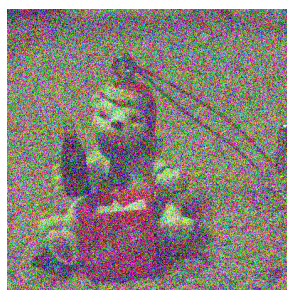

No-Flash Input

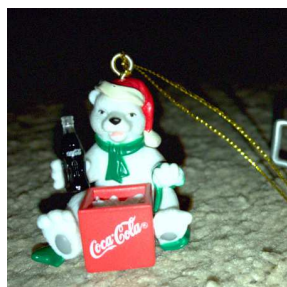

Flash Input

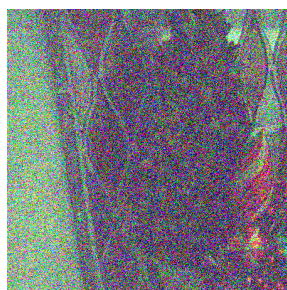

No-Flash Input

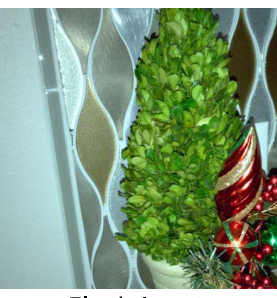

Flash Input

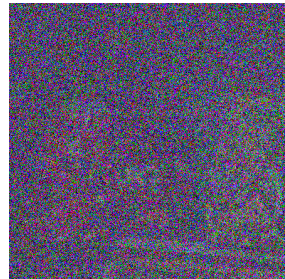

No-Flash Input

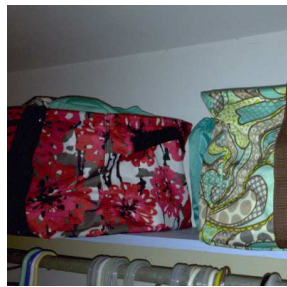

Flash Input

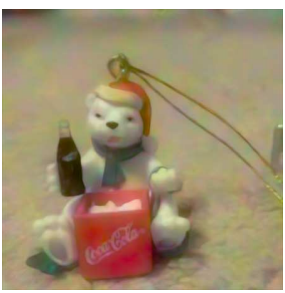

Our Filtered No-Flash

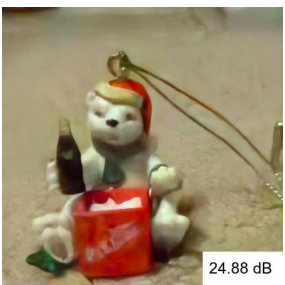

Only No-Flash Input

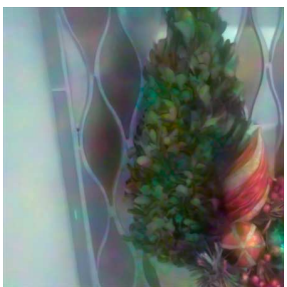

Our Filtered No-Flash

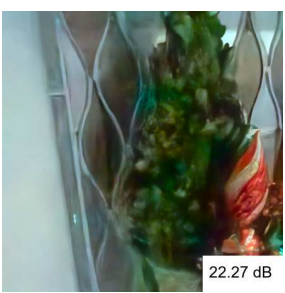

Only No-Flash Input

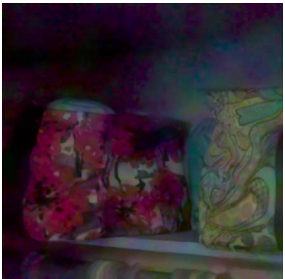

Our Filtered No-Flash

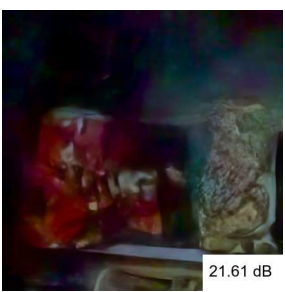

Only No-Flash Input

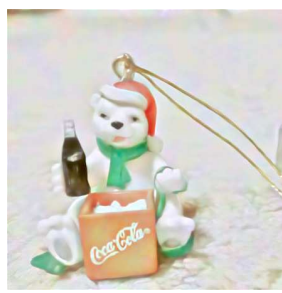

Our Scale Map

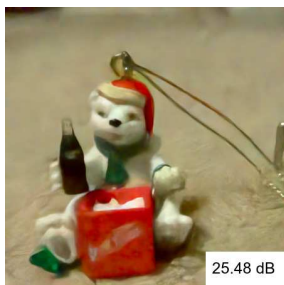

2x No-Flash Burst

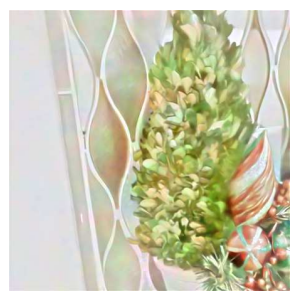

Our Scale Map

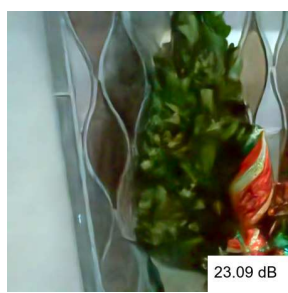

2x No-Flash Burst

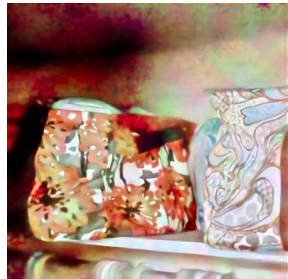

Our Scale Map

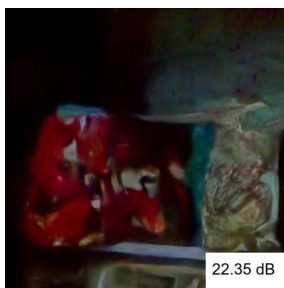

2x No-Flash Burst

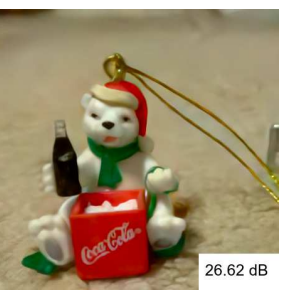

Our Final Output

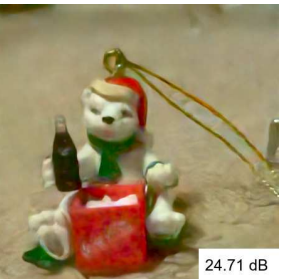

Direct $(\mathrm{F}+\mathrm{NF})$

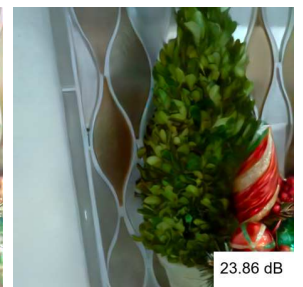

Our Final Output

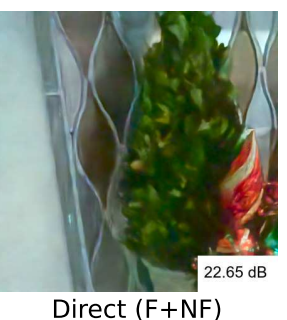

Direct $(\mathrm{F}+\mathrm{NF})$

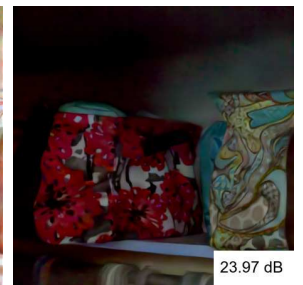

Our Final Output

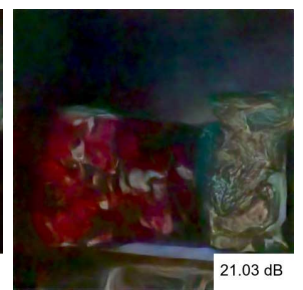

Direct $(\mathrm{F}+\mathrm{NF})$

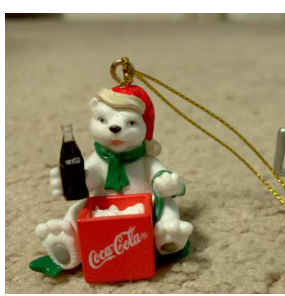

Ground-Truth

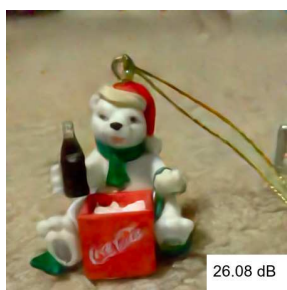

$\mathrm{BPN}(\mathrm{F}+\mathrm{NF})$

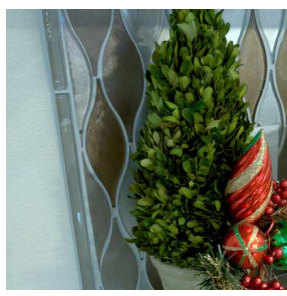

Ground-Truth

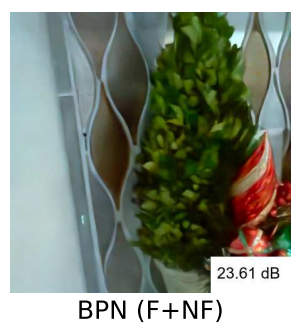

$\mathrm{BPN}(\mathrm{F}+\mathrm{NF})$

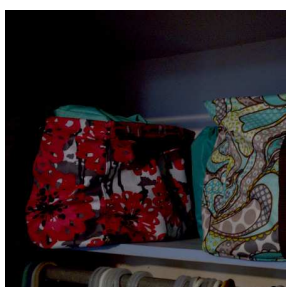

Ground-Truth

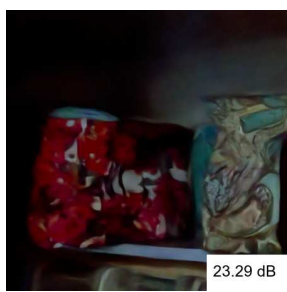

BPN $(F+N F)$

Figure 7: More qualitative comparisons. 


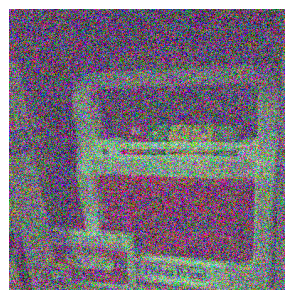

No-Flash Input

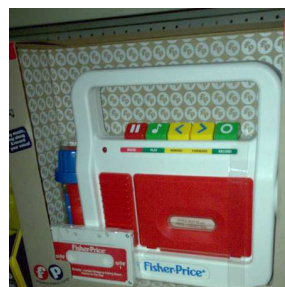

Flash Input

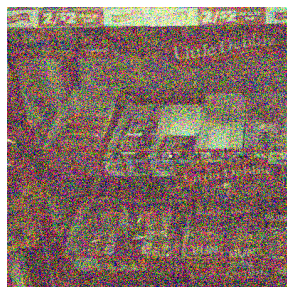

No-Flash Input

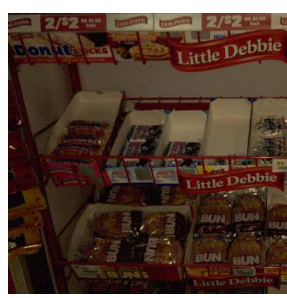

Flash Input

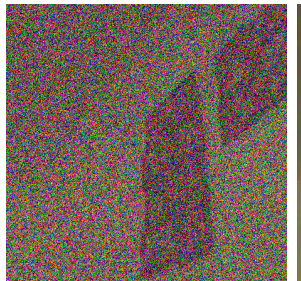

No-Flash Input

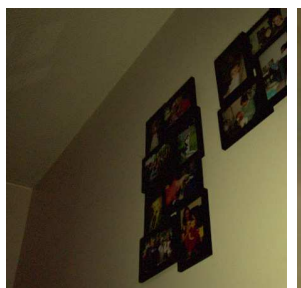

Flash Input

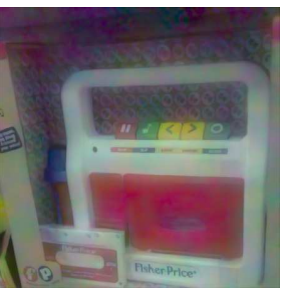

Our Filtered No-Flash
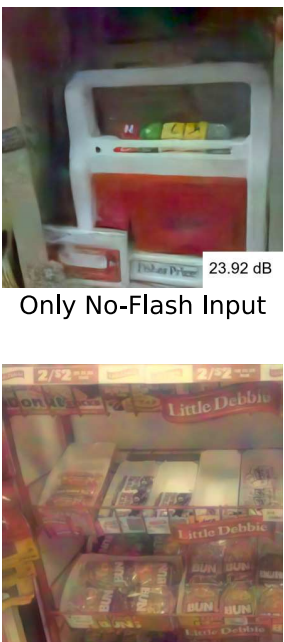

Our Filtered No-Flash
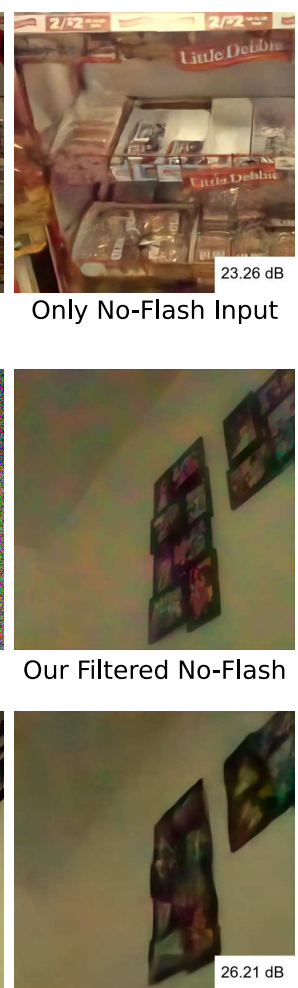

Only No-Flash Input

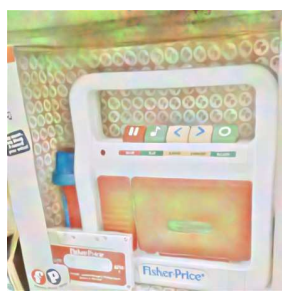

Our Scale Map

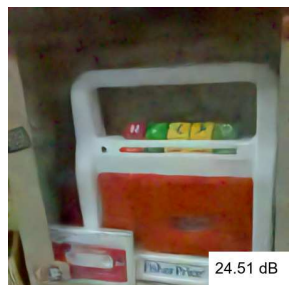

2x No-Flash Burst

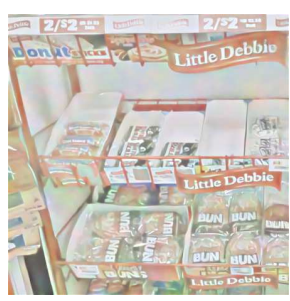

Our Scale Map

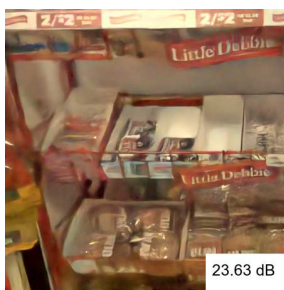

2x No-Flash Burst

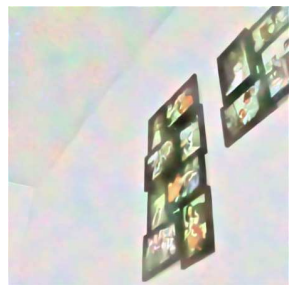

Our Scale Map

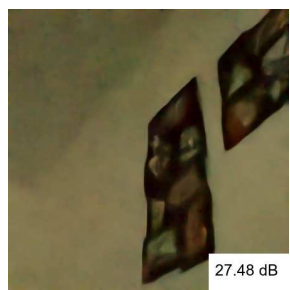

2x No-Flash Burst

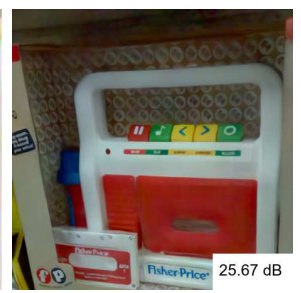

Our Final Output

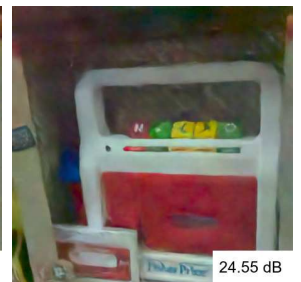

Direct $(\mathrm{F}+\mathrm{NF})$

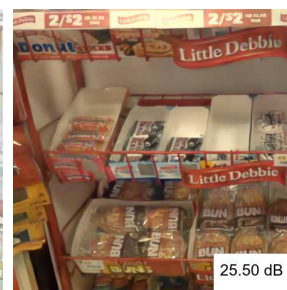

Our Final Output

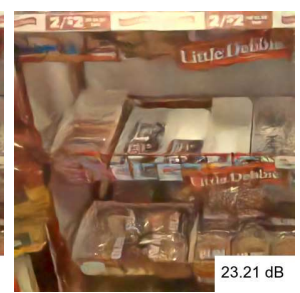

Direct $(\mathrm{F}+\mathrm{NF})$

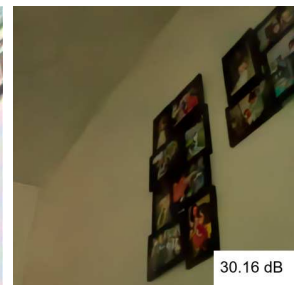

Our Final Output

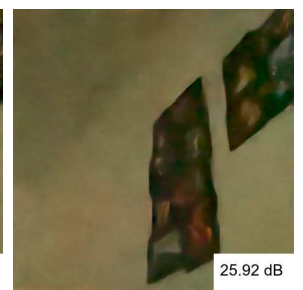

Direct $(\mathrm{F}+\mathrm{NF})$

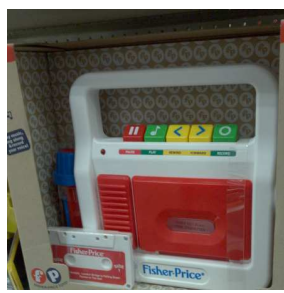

Ground-Truth

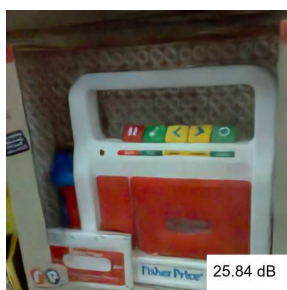

$\mathrm{BPN}(\mathrm{F}+\mathrm{NF})$

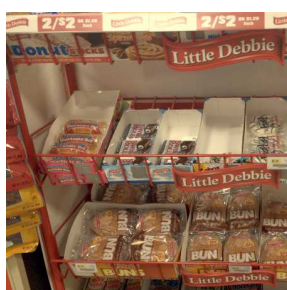

Ground-Truth

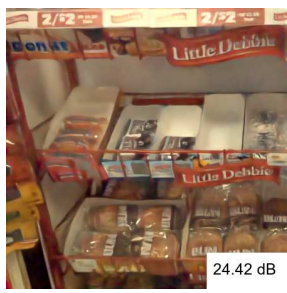

$\mathrm{BPN}(\mathrm{F}+\mathrm{NF})$

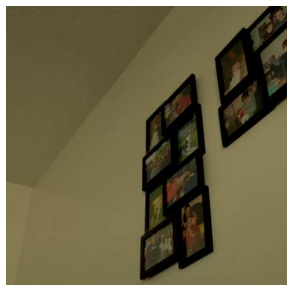

Ground-Truth

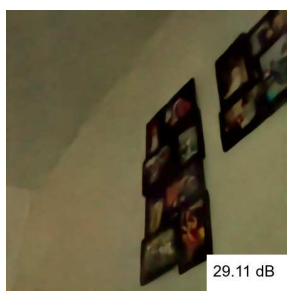

BPN $(F+N F)$

Figure 7: (continued) More qualitative comparisons. 


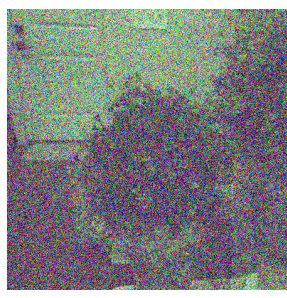

No-Flash Input

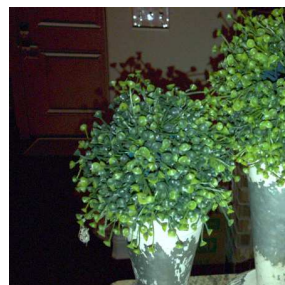

Flash Input

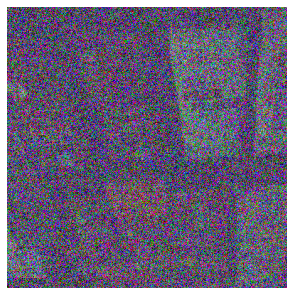

No-Flash Input

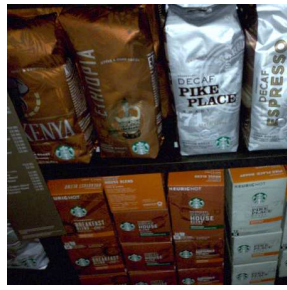

Flash Input

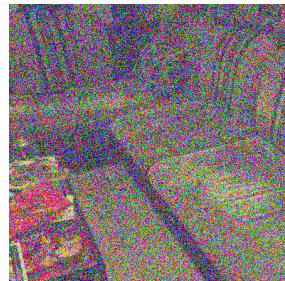

No-Flash Input

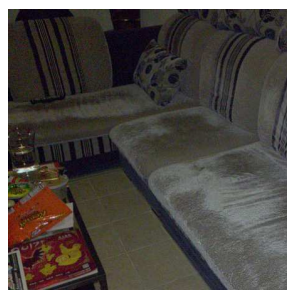

Flash Input
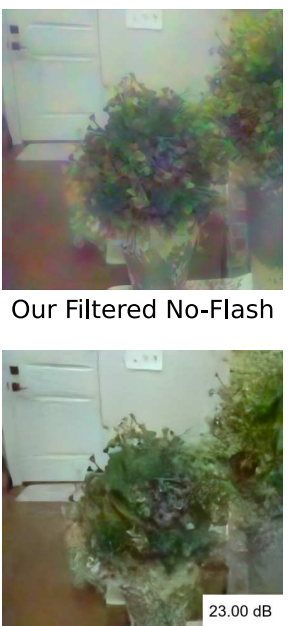

Only No-Flash Input

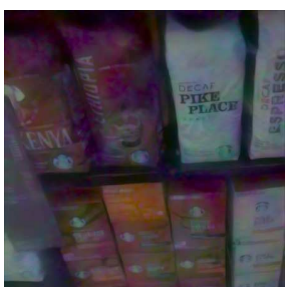

Our Filtered No-Flash

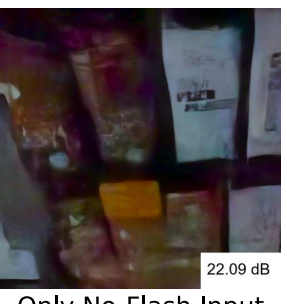

Only No-Flash Input

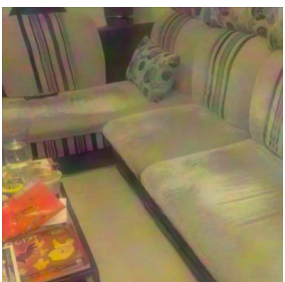

Our Filtered No-Flash

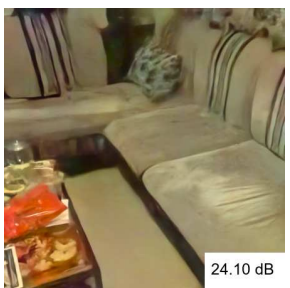

Only No-Flash Input

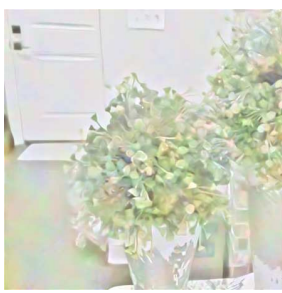

Our Scale Map

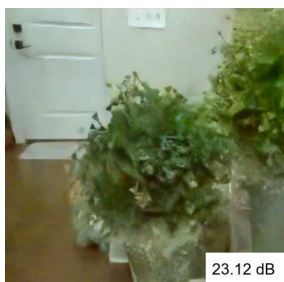

2x No-Flash Burst

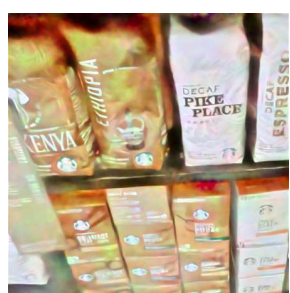

Our Scale Map

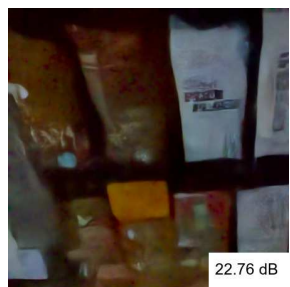

2x No-Flash Burst

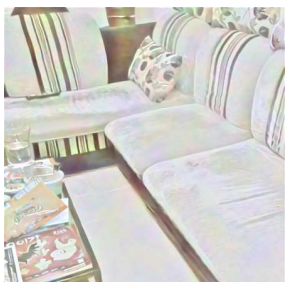

Our Scale Map

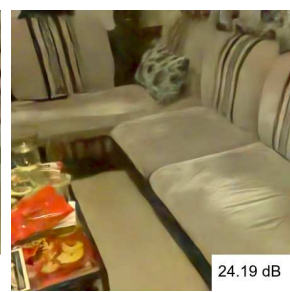

2x No-Flash Burst

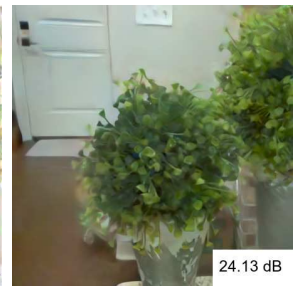

Our Final Output

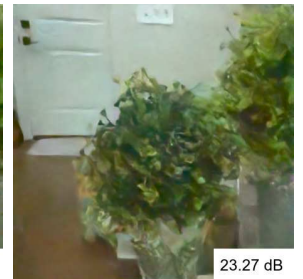

Direct $(\mathrm{F}+\mathrm{NF})$

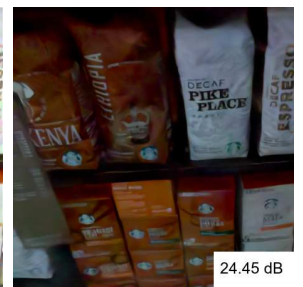

Our Final Output

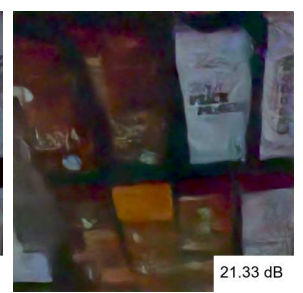

Direct $(\mathrm{F}+\mathrm{NF})$

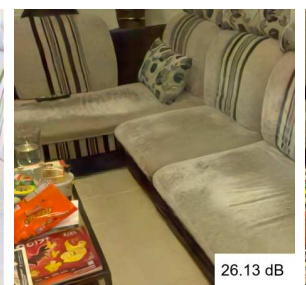

Our Final Output

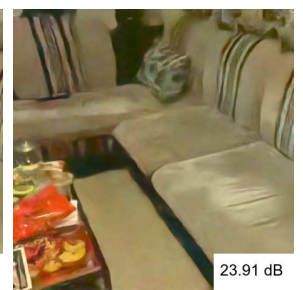

Direct $(\mathrm{F}+\mathrm{NF})$

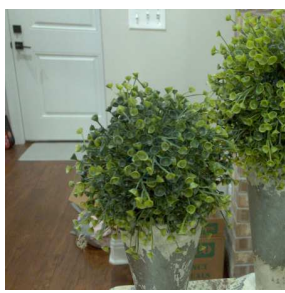

Ground-Truth

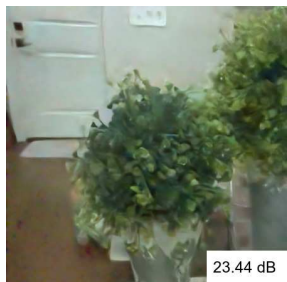

$\mathrm{BPN}(\mathrm{F}+\mathrm{NF})$

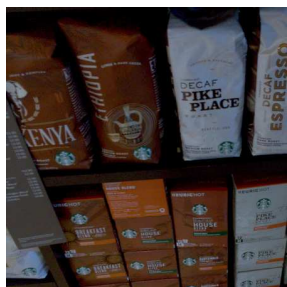

Ground-Truth

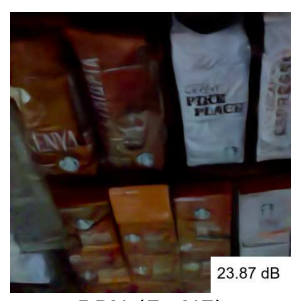

$\mathrm{BPN}(\mathrm{F}+\mathrm{NF})$

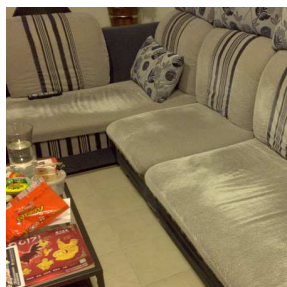

Ground-Truth

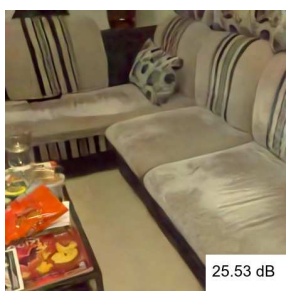

BPN $(F+N F)$

Figure 7: (continued) More qualitative comparisons. 


\begin{tabular}{lcccccccc}
\hline \multirow{2}{*}{ Method } & \multicolumn{2}{c}{ 100x Dimmed } & \multicolumn{2}{c}{ 50x Dimmed } & \multicolumn{2}{c}{ 25x Dimmed } & \multicolumn{2}{c}{ 12.5x Dimmed } \\
& PSNR & SSIM & PSNR & SSIM & PSNR & SSIM & PSNR & SSIM \\
\hline Direct Prediction & $24.15 \mathrm{~dB}$ & 0.779 & $26.00 \mathrm{~dB}$ & 0.810 & $27.31 \mathrm{~dB}$ & 0.836 & $28.17 \mathrm{~dB}$ & 0.856 \\
KPN [27] & $25.51 \mathrm{~dB}$ & 0.820 & $27.43 \mathrm{~dB}$ & 0.852 & $28.78 \mathrm{~dB}$ & 0.874 & $29.74 \mathrm{~dB}$ & 0.889 \\
BPN [35] & $26.23 \mathrm{~dB}$ & 0.831 & $27.83 \mathrm{~dB}$ & 0.857 & $29.08 \mathrm{~dB}$ & 0.877 & $30.00 \mathrm{~dB}$ & 0.891 \\
Ours & $\mathbf{2 6 . 8 3 ~ d B}$ & $\mathbf{0 . 8 4 3}$ & $\mathbf{2 8 . 3 9} \mathbf{d B}$ & $\mathbf{0 . 8 6 6}$ & $\mathbf{2 9 . 5 5} \mathbf{d B}$ & $\mathbf{0 . 8 8 3}$ & $\mathbf{3 0 . 4 5} \mathbf{d B}$ & $\mathbf{0 . 8 9 7}$ \\
\hline
\end{tabular}

Table 4: Quantitative Results using flash image as geometric reference for all methods.

\begin{tabular}{lcccccc}
\hline \multirow{2}{*}{ Noise Parameters } & \multicolumn{2}{c}{$\log \left(\left[\sigma_{r}, \sigma_{s}\right]\right)=[-2.8,-4.0]$} & \multicolumn{2}{c}{$\log \left(\left[\sigma_{r}, \sigma_{s}\right]\right)$} & $=[-2.4,-3.2]$ & \multicolumn{2}{c}{$\log \left(\left[\sigma_{r}, \sigma_{s}\right]\right)=[-2.2,-2.8]$} \\
& PSNR & SSIM & PSNR & SSIM & PSNR & SSIM \\
\hline Direct Prediction & $28.44 \mathrm{~dB}$ & 0.845 & $25.54 \mathrm{~dB}$ & 0.787 & $23.88 \mathrm{~dB}$ & 0.754 \\
KPN [27] & $29.01 \mathrm{~dB}$ & 0.870 & $26.82 \mathrm{~dB}$ & 0.832 & $25.59 \mathrm{~dB}$ & 0.808 \\
BPN [35] & $29.18 \mathrm{~dB}$ & 0.870 & $26.86 \mathrm{~dB}$ & 0.829 & $25.61 \mathrm{~dB}$ & 0.805 \\
Ours & $\mathbf{2 9 . 6 5 ~ d B}$ & $\mathbf{0 . 8 7 6}$ & $\mathbf{2 7 . 4 7} \mathbf{d B}$ & $\mathbf{0 . 8 4 2}$ & $\mathbf{2 6 . 2 6} \mathbf{d B}$ & $\mathbf{0 . 8 2 1}$ \\
\hline
\end{tabular}

Table 5: Noise levels. Performance of different approaches to denoising flash and no-flash pairs, at 50x dimmed light levels, with three additional noise levels (increasing from left to right). Note that the noise level used in the main results in the paper was between the first and second level above.

\begin{tabular}{lcccccccc}
\hline \multirow{2}{*}{ Method } & \multicolumn{2}{c}{ 100x Dimmed } & \multicolumn{2}{c}{ 50x Dimmed } & \multicolumn{2}{c}{ 25x Dimmed } & \multicolumn{2}{c}{ 12.5x Dimmed } \\
& PSNR & SSIM & PSNR & SSIM & PSNR & SSIM & PSNR & SSIM \\
\hline 2x No-flash (BPN [35]) & $25.58 \mathrm{~dB}$ & 0.796 & $27.75 \mathrm{~dB}$ & 0.839 & $29.65 \mathrm{~dB}$ & 0.874 & $31.21 \mathrm{~dB}$ & 0.899 \\
Flash and No-flash (Ours) & $26.75 \mathrm{~dB}$ & 0.829 & $28.56 \mathrm{~dB}$ & 0.860 & $30.14 \mathrm{~dB}$ & 0.884 & $31.52 \mathrm{~dB}$ & 0.903 \\
5x No-flash (BPN [35]) & $25.90 \mathrm{~dB}$ & 0.788 & $27.84 \mathrm{~dB}$ & 0.832 & $29.54 \mathrm{~dB}$ & 0.867 & $31.03 \mathrm{~dB}$ & 0.894 \\
Flash and 4x No-flash (Ours) & $26.82 \mathrm{~dB}$ & 0.822 & $28.60 \mathrm{~dB}$ & 0.856 & $30.21 \mathrm{~dB}$ & 0.883 & $31.60 \mathrm{~dB}$ & 0.904 \\
7x No-flash (BPN [35]) & $26.00 \mathrm{~dB}$ & 0.792 & $27.89 \mathrm{~dB}$ & 0.834 & $29.57 \mathrm{~dB}$ & 0.868 & $31.05 \mathrm{~dB}$ & 0.894 \\
Flash and 6x No-flash (Ours) & $26.80 \mathrm{~dB}$ & 0.820 & $28.60 \mathrm{~dB}$ & 0.855 & $30.20 \mathrm{~dB}$ & 0.882 & $31.59 \mathrm{~dB}$ & 0.903 \\
\hline
\end{tabular}

Table 6: Performance with larger bursts. We compare the original results of 2x no-flash burst with BPN [35] and flash and no-flash denoising with our method, to denoising larger bursts of no-flash images of length 5 and 7 with BPN, as well as using a modified version of our method with bursts of the same length where one of the images is captured with a flash.

Burst Denoising. We compared to using a burst of two no-flash images in the paper, as a means of evaluating the relative benefit of a the second image being taken with vs. without a flash. In both cases the second image provides additional information - a second no-flash image has high noise (though a different realization of noise than the first image), while a flash image has a much higher signal-to-noise ratio but entirely different shading. Our results showed that in this context, a second image is beneficial.

But more generally, burst photography (which typically involves a larger number of images) has its own relative strengths and weaknesses when compared to using flash and no-flash pairs, as a means of imaging in low-light. Burst denoising with longer bursts may well be a preferable option in the presence of moderate motion, or when using a flash is not an option (for example, when most objects in the scene are far away and can not be illuminated with a flash). Conversely, the use of a flash and no-flash pair is preferable in much lower light, in scenes where most of the scene can be well-illuminated with a flash, when camera or scene motion may cause significant misalignment across a large sequence of images, or when memory or computational constraints prevent capturing a larger sequence of images.

While the question of what acquisition strategy to use will depend on the environment and platform and is beyond the scope of this paper, we present a comparison in Table 6 to provide further intuition to the reader. We compare burst denoising, using BPN [35], with larger bursts of 5 and 7 images on our dataset-we use the same noise and dimming models to generate a larger burst of no-flash images. These sequences are mis-aligned using our randomly sampled homographies, with the homographies applied sequentially — thus the first and last image of a sequence will have a larger misalignment on average 

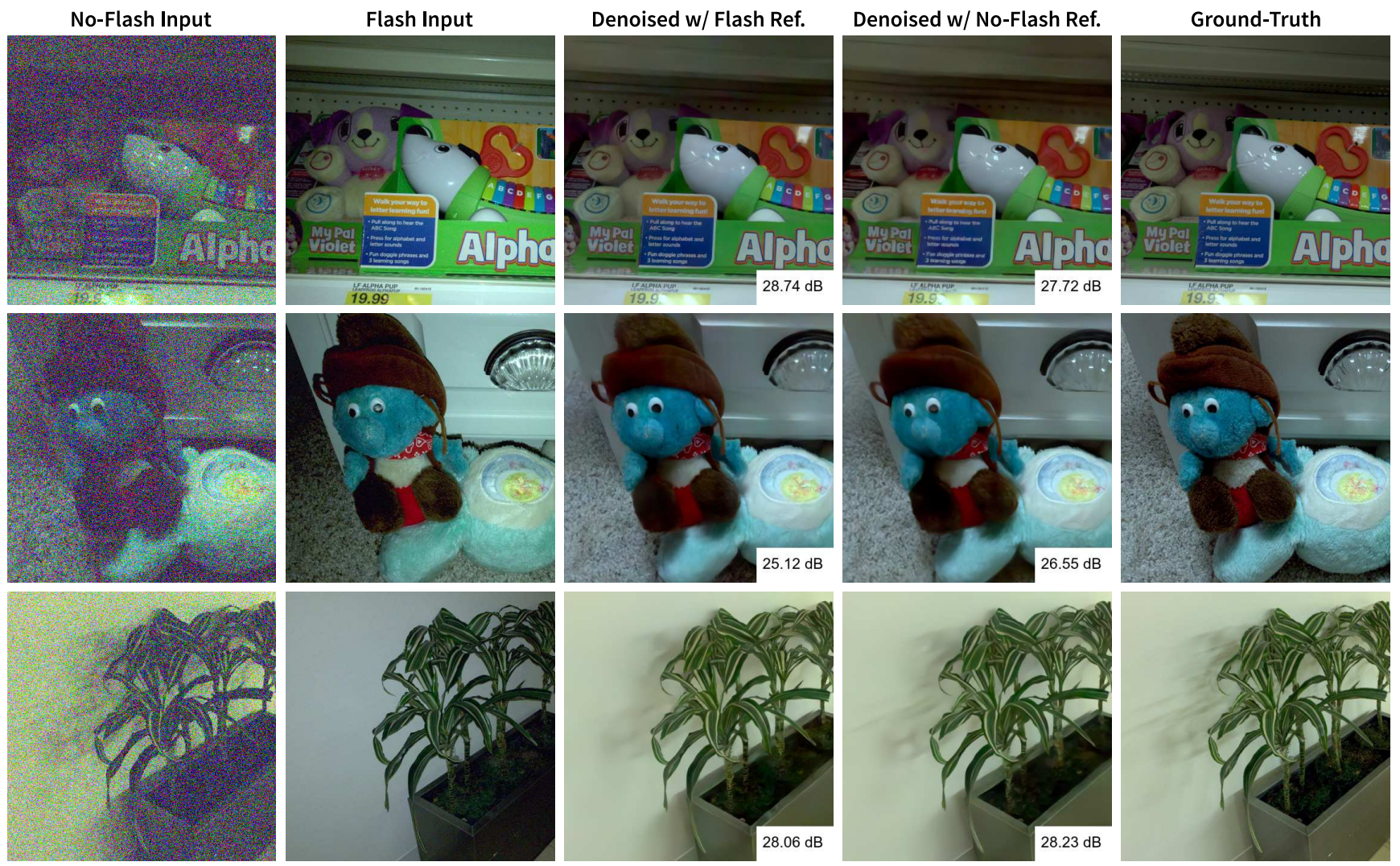

Figure 8: Flash vs No-Flash as reference. We show qualitative comparisons of results (at 50x dimmed light level) for our method using the flash input vs. the no-flash input as geometric reference. While using the no-flash input as reference does better on average, in some examples, using the flash as reference can lead to better reconstruction of high frequency detail (first row). In other cases, even though the flash-reference results are quantitatively, the difference is due to errors in reconstructing shading which are often less perceptually obvious. This is the case in the bottom two rows, although in the last row, we can see that the flash reference output has a blurrier reconstruction of the shadows on the wall.

than two subsequent pairs, and so we use the image in the middle of the sequence as reference. We compare these results to using our method when denoising bursts of the same size, where one (the last) image is taken with a flash and the rest without (again using the middle no-flash image as reference). Here, our network predicts kernels to be used to filter and sum all the no-flash images, which is then multiplied with our scale map. Because memory constraints, we do not use kernel upsampling in these experiments, and predict only a basis of $15 \times 15$ kernels (one for each channel of each no-flash image).

Our results show that in the light and motion settings we consider, larger no-flash bursts only have a modest improvement over a pair of two no-flash images at lower light levels, although they perform slightly worse comparatively at higher light levels (this is likely because the networks are trained over a range of light levels, and tend to oversmooth to handle the lowest end of that range). Our method, when using a burst of the same size with one as a flash image, performs better than pure noflash bursts, but also with only modest improvements over a flash and no-flash pair (note that in this case, the misalignment between the flash image and reference frame is greater than for a flash and no-flash pair that are taken in sequence). These results suggest that when using burst photography in settings where it is advantageous, it may be worth capturing one image of that burst with a flash. 